Published in final edited form as:

Cochrane Database Syst Rev. 2016 January 26; 2016(1): . doi:10.1002/14651858.CD012055.

\title{
Alternative agents versus prophylactic platelet transfusion for preventing bleeding in patients with thrombocytopenia due to chronic bone marrow failure: a network meta-analysis and systematic review
}

\author{
Michael Desborough ${ }^{1}$, Lise J Estcourt ${ }^{1}$, Anna Chaimani ${ }^{2}$, Carolyn Doree ${ }^{3}$, Sally Hopewell ${ }^{4}$, \\ Marialena Trivella ${ }^{5}$, Andreas V Hadjinicolaou ${ }^{6}$, Paresh Vyas ${ }^{7}$, and Simon J Stanworth ${ }^{8}$ \\ ${ }^{1}$ Haematology/Transfusion Medicine, NHS Blood and Transplant, Oxford, UK \\ ${ }^{2}$ Department of Hygiene and Epidemiology, University of loannina School of Medicine, loannina, \\ Greece \\ ${ }^{3}$ Systematic Review Initiative, NHS Blood and Transplant, Oxford, UK \\ ${ }^{4}$ Oxford Clinical Trials Research Unit, University of Oxford, Oxford, UK \\ ${ }^{5}$ Centre for Statistics in Medicine, University of Oxford, Oxford, UK \\ ${ }^{6}$ Human Immunology Unit, Institute of Molecular Medicine, Radcliffe Department of Medicine, \\ University of Oxford, Oxford, UK \\ ${ }^{7} \mathrm{MRC}$ Molecular Haematology Unit and Department of Haematology, University of Oxford and \\ Oxford University Hospitals NHS Trust, Oxford, UK \\ ${ }^{8}$ National Institute for Health Research (NIHR) Oxford Biomedical Research Centre, Oxford \\ University Hospitals NHS Foundation Trust and the University of Oxford, Oxford, UK
}

\begin{abstract}
This is the protocol for a review and there is no abstract. The objectives are as follows:

To compare the relative efficacy of different treatments for thrombocytopenia (artificial platelet substitutes, platelet-poor plasma, fibrinogen, rFVIIa, rFXIII, thrombopoietin mimetics, antifibrinolytic drugs or platelet transfusions) in patients with chronic bone marrow failure and to derive a hierarchy of potential alternate treatments to platelet transfusions.
\end{abstract}

\section{BACKGROUND}

\section{Description of the condition}

The bone marrow is the production site for red blood cells, white blood cells and platelets from stem cells, during the processes termed collectively as haematopoiesis. Bone marrow

Contact address: Michael Desborough, Haematology/Transfusion Medicine, NHS Blood and Transplant, Oxford, UK. michael.desborough@ouh.nhs.uk.

Editorial group: Cochrane Haematological Malignancies Group.

Publication status and date: Edited (no change to conclusions), published in Issue 2, 2016. 
failure disorders encompass a wide range of diseases that cause quantitative (reduced numbers) or qualitative (reduced function) defects of red cells, white cells and platelets.

Clinical symptoms of patients with bone marrow failure disorders are related to cytopenia, that is, the failure to produce adequate numbers of normal red cells, white cells, or platelets. Patients can present with fatigue and shortness of breath due to anaemia, recurrent infections due to neutropenia, and bleeding or bruising due to thrombocytopenia. Bleeding is a result of a failure to produce adequate numbers of platelets because of insufficient numbers of bone marrow megakaryocytes (cells in the bone marrow that produce platelets) or megakaryocyte dysfunction. Bone marrow failure disorders can also be associated with an increased risk of progression to acute leukaemia.

Bone marrow failure disorders can be classified according to the underlying pathophysiology, into four broad categories: myelodysplastic syndromes (MDS), primary myelofibrosis, acquired aplastic anaemia, and inherited bone marrow failure disorders.

MDS encompasses a diverse group of disorders that are characterised by dysplasia in one or more cell lines (blood cells have an abnormal shape or size), ineffective haematopoiesis, and an increased risk of developing acute myeloid leukaemia (AML). Overall the incidence of MDS is estimated at between 2.3 to 4.5 per 100,000 per year; however, incidence increases markedly with age, peaking in those aged over 80 years (> 30 per 100,000 per year) (Dinmohamed 2014; Ma 2007; Ma 2012; Neukirchen 2011). Several cohort studies have evaluated incidence of thrombocytopenia at diagnosis (platelet count $<100 \times 10^{9} / \mathrm{L}$ ), which affects $23 \%$ to $93 \%$ of patients with newly diagnosed MDS,depending on the cohort (Kantarjian 2007). Cohort studies report that haemorrhage is the cause of death in 14\% to $24 \%$ cases of MDS (Foucar 1985; Gupta 1999; Kantarjian 2007; Konstantopoulos 1989; Lidbeck 1980).

Primary myelofibrosis is a clonal myeloproliferative disease whereby the normal bone marrow is replaced by fibrosis, resulting in bone marrow failure. It has an incidence of 2.2 to 9.9 per million per year (Titmarsh 2014). Patients may develop a number of symptoms including fatigue, sweats, fevers, weight loss and an enlarged spleen, as well as symptoms of bone marrow failure (Tefferi 2013).

Acquired aplastic anaemia is a disease that results in a hypocellular bone marrow with quantitative defects of all three cell lines. The incidence in Europe and North America is two per million population per year and has a biphasic age distribution with increasing numbers of cases in those aged 10 to 25 years and those over 60 years (Heimpel 2000; Issaragrisil 2006; Montané 2008). The incidence in Asia is higher, with estimates ranging from 3.9 to 7.4 per million per year (Young 2008). The underlying cause of aplastic anaemia is unknown in most cases, but different reports have associated it with certain industrial chemicals (Young 2008), agricultural pesticides (Issaragrisil 2006; Muir 2003), drugs (Issaragrisil 2006; Young 2008), and hepatitis viruses (Rauff 2011).

Inherited bone marrow failure disorders that result in thrombocytopenia include those associated with a global haematopoietic defect such as Fanconi anaemia, Dyskeratosis congenita, or Swachman-Diamond syndrome, as well as disorders associated with isolated 
thrombocytopenia, such as thrombocytopenia with absent radii (TAR) and amegakaryocytic thrombocytopenia (Alter 2007). The most common inherited bone marrow disorder is Fanconi anaemia, which has a reported incidence of approximately 1 in 360,000 live births, with a carrier frequency of 1 in 300 (Swift 1971).

Treatment is tailored to the needs of individual patients but may include intensive treatment with allogeneic stem cell transplantation (Dokal 2008). Other patients are managed symptomatically, with low dose chemotherapy, or in the case of aplastic anaemia, with immunosuppressive agents, with a focus on maintaining quality of life, prolonging life and delaying transformation to acute leukaemia.

\section{Description of the intervention}

Platelet transfusions are of some benefit in managing active bleeding for patients with bone marrow failure and severe thrombocytopenia. The standard practice in most haematology units across the developed world is to use prophylactic transfusions in line with guidelines (BCSH 2003; BCSH 2004; NBA 2012; Schiffer 2001; Slichter 2007; Tinmouth 2007). For chronic bone marrow failure, prophylactic platelet transfusions are standard for patients with a platelet count less than $10 \times 10^{9} / \mathrm{L}$ and a haemorrhagic phenotype, but for patients without a haemorrhagic phenotype, platelet transfusions are not given. It is still uncertain how best to use platelet transfusions to prevent severe and life-threatening bleeding (Estcourt 2011). Alternatives to platelets may be more effective than platelet transfusions at controlling bleeding and will have a different side effect profile. Alternatives include artificial platelet substitutes, platelet-poor plasma, recombinant factor VIIa (rFVIIa), fibrinogen, recombinant factor XIII (rFXIII), thrombopoietin mimetics and antifibrinolytic drugs.

\section{How the intervention might work}

In normal haemostasis (formation of a blood clot), platelets form a primary haemostatic plug that is consolidated by the deposition of cross-linked fibrin. Platelet adhesion depends on normal platelet function, the presence of von Willebrand factor, and extracellular matrix components such as collagen and fibronectin (Ruggeri 2007). When the platelet count is low, the standard treatment has been to transfuse platelets, although this procedure can be associated with hazards such as infection, transfusion reactions and formation of antiplatelet antibodies. Additionally, $18 \%$ to $23 \%$ patients with bone marrow failure due to myelodysplastic syndrome have a haemorrhagic phenotype regardless of their platelet count (Kantarjian 2007). Alternatives to platelet transfusion aim to either simulate the effects of platelets (artificial platelet substitutes), stimulate additional fibrin formation (platelet poor plasma, recombinant factor VIIa and fibrinogen), promote von Willebrand factor release and platelet function (desmopressin), or increase platelet production (TPO mimetics). These agents aim to promote haemostasis without the side effects associated with platelet transfusions. The important adverse event for any pro-haemostatic intervention is thrombosis, and any of these interventions have the potential to cause it. Other specific adverse events are listed below with the description of the intervention.

Artificial platelet substitutes-Artificial platelet substitutes such as microspheres of human albumin coated with fibrinogen. lyophilised platelets, infusible plasma membranes; 
and liposomes with inserted platelet receptors aim to reproduce the active components of platelets without associated adverse events (Roberts 2013).

Platelet-poor plasma (PPP)_-Platelet-poor plasma (PPP) is a source of clotting factors and fibrinogen and can be administered intravenously. PPP is a blood component and is associated with a small risk of transfusion reactions and transfusion-transmitted infections (Desborough 2012).

Recombinant factor VIla (rFVIla)—Recombinant factor VIIa (rFVIIa) is an intravenous drug licensed for patients with haemophilia and inhibitory allo-antibodies, and for prophylaxis and treatment of patients with congenital factor VII deficiency. It is used offlicense in a number of other settings, including operations where blood loss cannot be controlled by other means. However, the effectiveness of its use in patients without haemophilia is unproven (Simpson 2012).

Fibrinogen concentrate-The final step of the coagulation cascade is the formation of a fibrin clot. The substrate for fibrin is fibrinogen, which is converted into fibrin by the action of thrombin. Fibrinogen concentrate is administered intravenously and may result in some reduction in surgical bleeding when administered pre-operatively, although the overall quality of evidence for this is low (Wikkelsø 2013). Fibrinogen concentrate is a blood component and is associated with a theoretical risk of viral infection. However, viral inactivation is involved in its manufacture and is likely to make this risk very low (Franchini 2012).

Recombinant factor XIII (rFXIII)-In a normal clot, when single strands of fibrin have been formed, they are cross-linked by factor XIII, giving the clot strength. Trials of rFXIII have taken place in patients undergoing cardiac surgery to assess whether this reduced postsurgical bleeding (Karkouti 2013).

Desmopressin (DDAVP)—Desmopressin (DDAVP) is a vasopressin analogue that increases the plasma levels of factor VIII (FVIII) and von Willebrand factor (vWF) two- to three-fold. It is used to treat patients with mild haemophilia A or von Willebrand disease and has also been used to treat patients with uraemia, liver cirrhosis, congenital platelet function disorders and drug-induced platelet dysfunction (Svensson 2014). It can be administered intravenously, subcutaneously or intranasally. These different routes of administration result in different levels of vWF and factor VIII response (Mannucci 1987). If we include trials comparing more than one route of administration of DDAVP, then we will perform sensitivity analyses to determine if they can be combined as a single node. DDAVP is a welltolerated medication, but it is associated with facial flushing and can potentially cause hyponatraemic seizures in patients who are not fluid-restricted (Svensson 2014).

Thrombopoietin (TPO) mimetics-The liver synthesises thrombopoietin (TPO), which is the key regulator of bone marrow platelet production. TPO mimetics have been used in several disease states to promote both megakaryopoiesis and thrombopoiesis (Kuter 2014). The two main TPO mimetics in current use are romiplostim (weekly injection) and eltrombopag (daily oral tablet), both of which are recommended by the National Institute for 
Health and Care Excellence (NICE) for use in adults with immune thrombocytopenia (ITP) who have severe disease and a high risk of bleeding (NICE 2011; NICE 2013). While a systematic review found that these agents improve platelet counts, there was no evidence that TPO receptor agonists reduced the risk of significant bleeding for patients with ITP (Zeng 2011). Interleukin 6 and interleukin 11 may also act as stimulants of thrombopoiesis (Gordon 1995; Kurzrock 2001; Tsimberidou 2005).

Antifibrinolytic drugs-Fibrinolysis is the process by which blood clots are broken down after they have been formed. Anti-fibrinolytic drugs block this process, resulting in greater clot strength. The three most commonly used antifibrinolytic drugs are tranexamic acid, aprotinin and epsilon-aminocaproic acid. A previous systematic review assessed these agents (Wardrop 2013), which are included in this review for comparison with other potential interventions.

\section{Why it is important to do this review}

This review will focus on the question of whether alternative agents to prophylactic platelet transfusions are effective for the prevention and control of life-threatening thrombocytopenic bleeding. Platelet transfusions are expensive and may lead to adverse events such as infections and platelet refractoriness, particularly in groups of patients who receive multiple transfusions, such as those with chronic bone marrow failure. Some patients with bone marrow failure bleed despite apparently adequate platelet numbers, and alternative methods for managing bleeding will be necessary. This review will also be important for the developing world, where access to safe blood components is much more limited (Verma 2009). It is unlikely that there will be any head-to-head trials of the interventions in this review, and this leaves uncertainty for decision-makers.

\section{OBJECTIVES}

To compare the relative efficacy of different treatments for thrombocytopenia (artificial platelet substitutes, platelet-poor plasma, fibrinogen, rFVIIa, rFXIII, thrombopoietin mimetics, antifibrinolytic drugs or platelet transfusions) in patients with chronic bone marrow failure and to derive a hierarchy of potential alternate treatments to platelet transfusions.

\section{METHODS}

\section{Criteria for considering studies for this review}

Types of studies-We will include only randomised controlled trials (RCTs).

Types of participants-Our review will include inpatients and outpatients of all ages with thrombocytopenia due to chronic bone marrow failure. If trials consist of mixed populations of patients (e.g. those with diagnoses of immune thrombocytopenic purpura), we will use only data from the bone marrow failure subgroups. If subgroup data for patients with bone marrow failure is not available (even after contacting the authors of the trial), we will exclude the trial if fewer than $80 \%$ of participants have bone marrow failure. We will take particular care to ensure that transitivity is not violated when subgroups are included in 
the analysis. We will exclude any participants that do not have thrombocytopenia due to bone marrow failure, as well as participants undergoing intensive chemotherapy or stem cell transplantation, as this is the focus of another review (Estcourt 2014). We will include patients with bone marrow failure syndrome (e.g. aplastic anaemia, congenital bone marrow failure syndromes, myelodysplastic syndrome and myelofibrosis) that are not being treated with intensive chemotherapy or an allogeneic stem cell transplant.

Types of interventions-It is unlikely that any studies will have directly compared some of the alternative interventions. In particular, the two main licensed TPO mimetics (eltrombopag and romipostim) are manufactured by different drug companies (GlaxoSmithKline and Amgen, respectively), so it is unlikely that any studies comparing them exist. Consequently, we plan to perform a network meta-analysis to rank each of the interventions according to their efficacy in the control of bleeding to aid clinical decisionmaking. We will include each possible pairwise comparison (Figure 1), along with any other eligible interventions, which may not be of direct interest but could shed light on the effect of other included agents (Ades 2013). It is likely that the majority of trials will be of TPO mimetics, and we will evaluate romiplostim and eltrombopag separately. If trials include more than one dose of each intervention, then we will assess whether the different doses are equivalent. If we can reasonably consider the doses of an intervention to be equivalent, we will combine them into a single node in the network; otherwise we will analyse them separately. If applicable, we will perform a sensitivity analysis to compare possible assumptions about the different doses (Del Giovane 2013).

Interventions of direct interest: We will consider the following interventions (alternative agents) without restrictions on the dose.

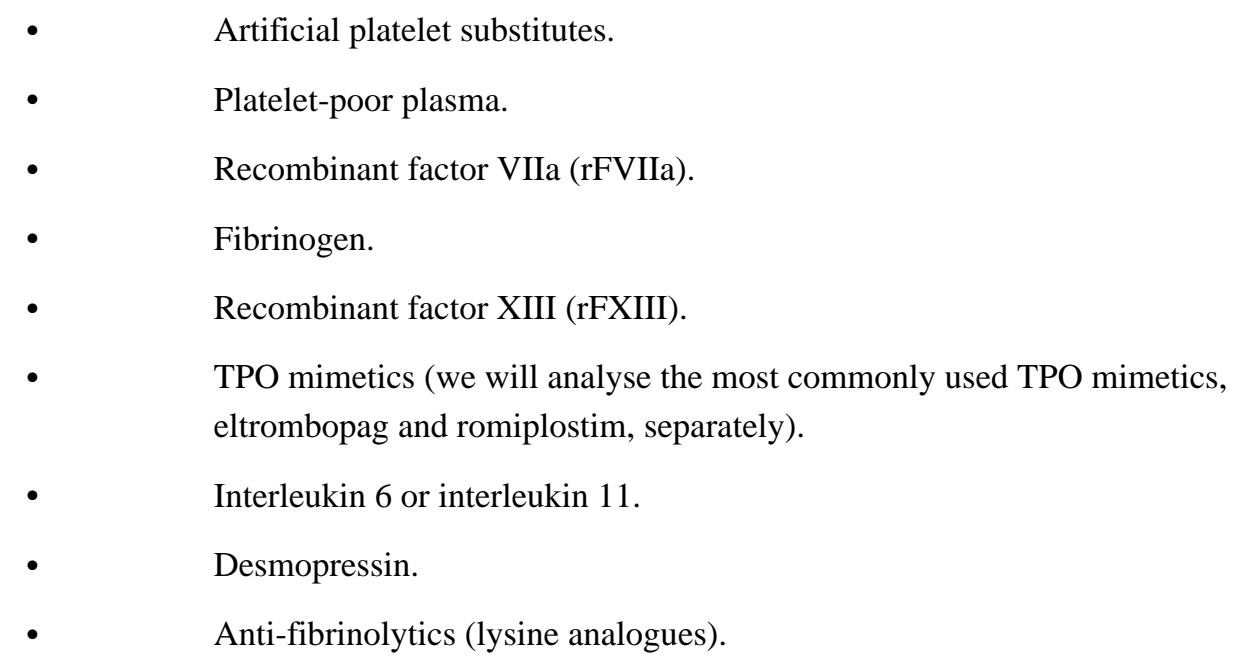

Inclusion of additional interventions to supplement the analysis: We will include randomised controlled trials that evaluate one or more of the interventions listed above. If we identify interventions we are not aware of, we will consider them as eligible and include them in the network analysis after assessing their comparability with the pre-specified set of 
competing interventions. We will report the findings for all interventions of direct interest in the results and conclusions of the review.

For the purposes of this analysis, we assume that any patient that meets the inclusion criteria is, in principle, equally likely to be randomised to any of the eligible interventions (Salanti 2012).

Types of outcome measures-We will categorise all outcomes according to short-, medium-, and long-term outcomes. Studies that meet the other inclusion criteria will be included in this review regardless of whether they include these outcomes. We will report the exact definition of these time frames over time periods that are common to as many studies as possible (e.g. up to 30 days, one to six months, and greater than six months from day of randomisation). We will use the primary outcomes and adverse events to develop a hierarchy of treatments.

\section{Primary outcomes}

1. Number of patients with at least one bleeding episode

2. Number of patients with at least one severe or life-threatening bleeding episode

3. Number of days bleeding occurred per patient

\section{Secondary outcomes}

1.

Mortality

i) Overall mortality

ii) Mortality due to bleeding

iii) Mortality due to infection

2.

Platelet transfusions

i) Proportion of patients requiring a platelet transfusion

ii) Number of units of platelets transfused per patient

iii) Mean number of platelet transfusion episodes per patient

3. Red cell transfusions

i) Proportion of patients requiring a red cell transfusion

ii) Number of units of red cells transfused per patient

iii) Number of red cell transfusion episodes per patient

4. Adverse events (e.g. transfusion reactions, transfusion-transmitted infections, thromboembolism, development of platelet antibodies, development of platelet refractoriness, drug reactions) 


\section{Search methods for identification of studies}

The Systematic Review Initiative (SRI) Information Specialist (CD) will formulate the search strategies in collaboration with the Cochrane Haematological Malignancies Group. The search will include all possible comparisons formed by the interventions of interest.

\section{Electronic searches}

Bibliographic databases: We will search for randomised controlled trials in the following databases.

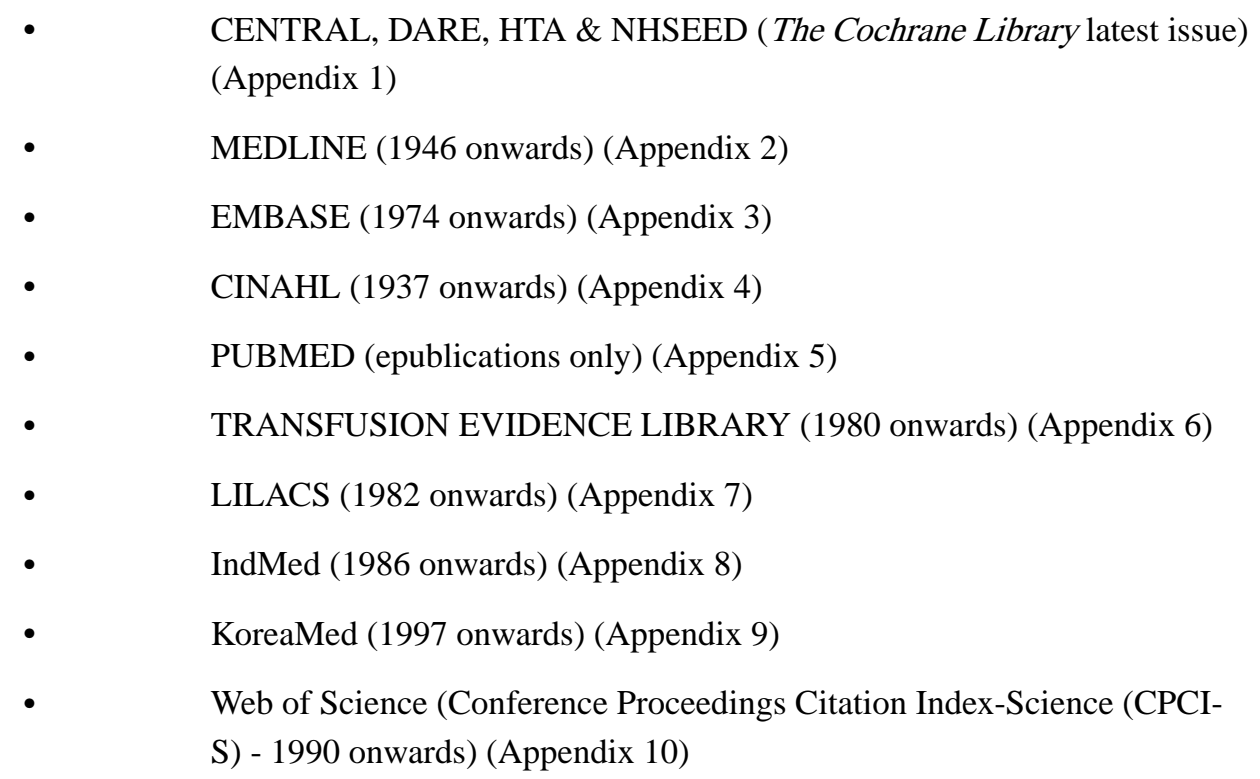

We will combine searches in MEDLINE, EMBASE and CINAHL with adaptations of the Cochrane RCT search filters, as described in Chapter 6 of the Cochrane Handbook for Systematic Reviews of Interventions (Lefebvre 2011). We will combine searches in CINAHL with the relevant SIGN RCT studies filter (www.sign.ac.uk/methodology/ filters.html). We present all search strategies in the appendices as indicated. There will be no restrictions on language or publication status.

Ongoing studies: We will identify ongoing trials with searches of ClinicalTrials.gov (http:// clinicaltrials.gov/ct2/search) (Appendix 11), the WHO International Clinical Trials Registry Platform (ICTRP) (http://apps.who.int/trialsearch/) (Appendix 12) and the Hong Kong Clinical Trials Registry (http://www.hkclinicaltrials.com/) (Appendix 13).

Searching other resources-We will handsearch references of all included trials, relevant review articles, and current treatment guidelines for further literature, limiting these searches to the 'first generation' reference lists. We will also seek information about ongoing trials and unpublished data from study authors and sponsors.

\section{Data collection and analysis}

Selection of studies-We will select studies according to the methods described in Chapter 7 of the Cochrane Handbook for Systematic Reviews of Interventions (Higgins 
2011a). Two independent review authors (MD, AH) will initially screen all electronically derived citations and abstracts of papers identified by the review search strategy for relevance. We will exclude clearly irrelevant studies at this stage. The same two review authors will then independently assess the full texts of all potentially relevant trials for eligibility against the criteria outlined above. In case of disagreements, a third review author (SS) will act as adjudicator. We will request further information from study authors if the article contains insufficient data to make a decision about eligibility. To assess the relevance of trials on platelet transfusion, we will design a study eligibility form, which will help ascertain whether the participants had thrombocytopenia due to bone marrow failure and whether trial arms differed according to their use of an alternative agent to prophylactic platelet transfusions. We will record the reasons why potentially relevant studies failed to meet the eligibility criteria and display the results of the search in a PRISMA flow chart (Hutton 2015).

Data extraction and management-Two review authors (MD, AH) will conduct data extraction according to the guidelines proposed by Cochrane (Higgins 2011a). We will resolve potential disagreements between the review authors by consensus. The review authors will not be blinded to names of authors, institutions, journals, or the outcomes of the trials. A related review team has already piloted the data extraction forms (Estcourt 2014). The two authors (MD, $\mathrm{AH}$ ) will independently extract the following data for all the studies.

- General information: review author's name, date of data extraction, study ID, reference manager number, first author of study, author's contact address (if available), citation of paper and objectives of the trial.

-

Trial details: trial design, location, setting, sample size, power calculation, treatment allocation, randomisation, blinding, inclusion and exclusion criteria, reasons for exclusion, comparability of groups, length of follow-up, stratification, stopping rules described, statistical analysis, results, conclusion and funding.

- Characteristics of participants: age, sex, ethnicity, total number recruited, total number randomised, total number analysed, types of bone marrow failure, severity of disease, baseline platelet count, numbers lost to follow-up, drop-outs (percentage in each arm) with reasons, protocol violations, previous treatments, current treatment and prognostic factors. We will use the type of bone marrow failure, severity of disease and baseline platelet count for the evaluation of the transitivity assumption (Jansen 2013; Salanti 2012).

- Characteristics of interventions: number of study arms, description of experimental arm(s), description of control arm, type of platelet transfusion given, timing of intervention, dosage of platelet given, compliance to interventions, additional interventions given especially in relation to red cell transfusions and any other differences between interventions. 
- $\quad$ Outcomes: number and severity of bleeding episodes, mortality (all causes), mortality due to bleeding, mortality due to infection, mean number of platelet and red cell transfusions, proportion of patients requiring each type of transfusion and adverse events (e.g. transfusion reactions, transfusion-transmitted infections, thromboembolism, development of platelet antibodies, development of platelet refractoriness, drug reactions). We will use both full-text versions and abstracts to retrieve the data. We will extract arm-level data rather than study-level data. For publications reporting on more than one trial, we will use one data extraction form for each trial. If these sources do not provide sufficient information, we will contact the authors, study groups or companies for additional details. One review author (MD) will enter data into software, and another $(\mathrm{AH})$ will check data entry for accuracy.

Data on potential effect modifiers: For each individual study, we will extract data on the following study, intervention and population characteristics that may act as modifiers.

- $\quad$ Cause of bone marrow failure.

- Severity of disease.

- $\quad$ Baseline platelet count.

- Concurrent medications.

Assessment of risk of bias in included studies-We will assess the quality of all RCTs using the Cochrane 'Risk of bias' criteria, as described in Chapter 8 of the Cochrane Handbook for Systematic Reviews of Interventions (Higgins 2011b).Two review authors (MD, AH) will work independently to assess each element of potential bias listed below as carrying a high, low or unclear risk. We will briefly describe the judgement statements upon which reviewers have assessed potential bias in the 'Characteristics of included studies' table. We will reach a consensus on the degree of risk of bias by comparing the review authors' statements and where necessary, through consultation with a third author (SS). We will use the 'Risk of bias' assessment to explore statistical heterogeneity in each included study and to perform sensitivity analyses. We will use Cochrane's tool for assessing risk of bias (low, high or unclear risk) in the following areas.

- $\quad$ Selection bias (random sequence generation and allocation concealment).

- $\quad$ Performance bias (blinding of participants and personnel).

- Detection bias (blinding of outcome assessment).

- $\quad$ Attrition bias (incomplete outcome data).

- $\quad$ Reporting bias (selective reporting).

- $\quad$ Other bias.

We will assess risk of bias separately for each key outcome of the review. 
Measures of treatment effect-For dichotomous outcomes (number of patients with at least one bleeding episode, number of patients with at least one severe or life-threatening bleeding episode, overall mortality, mortality due to bleeding, mortality due to infection, proportion of patients requiring a platelet transfusion, proportion of patients requiring a red cell transfusion, adverse events), we will record the number of events and the total number of participants in both the treatment and control groups.

For continuous outcomes (number of days bleeding occurred per patient, number of units of platelets transfused per patient, mean number of platelet transfusion episodes per patient, number of units of red cells transfused per patient, number of red cell transfusion episodes per patient), we will record the mean, standard deviation and total number of participants in both the treatment and control groups. For studies providing only study-level data, we will extract the reported effect size with the corresponding standard error. If data allow we will undertake quantitative assessments using Stata (StataCorp 2011).

We will analyse continuous outcomes measured using the same scale, using the mean difference (MD) with 95\% confidence intervals (CIs). For continuous outcomes measured with different scales, we will present the standardised mean difference (SMD).

Relative treatment effects: For time to-event outcomes such as overall survival, we will extract the hazard ratio (HR) from published data. If HRs are not available, we will make every effort to estimate as accurately as possible the HR using the available data and a purpose-built method based on the Parmar and Tierney tool (Parmar 1998; Tierney 2007). For dichotomous outcomes, we will report odds ratios (ORs) with a 95\% CI (and for mortality, if HRs are not available). If appropriate, we will report the number needed to treat for an additional beneficial outcome (NNTB) and the number needed to treat for an additional harmful outcome (NNTH) along with their CIs. If we cannot report the available data in any of the formats described above, we will provide a descriptive summary of the available information.

We will estimate the pairwise relative treatment effects of the competing interventions using the proportion of patients with significant bleeding, the proportion of patients with an adverse events and the proportion of patients requiring a platelet transfusion. We will then analyse these dichotomous outcomes by calculating an odds ratio.

Relative treatment ranking: We will obtain a hierarchy of the competing interventions using the surface area under the cumulative ranking curve (SUCRA) for the primary outcomes and the adverse events (Salanti 2011).

\section{Unit of analysis issues}

Cross-over trials: For cross-over trials, we will not assess the long-term outcomes mortality and proportion of patients in complete remission. We will assess other outcomes if the timing of the outcome measure occurs before the cross-over and if outcomes after the crossover are not biased by the treatment before the cross-over. We will examine each trial individually to determine this eventuality. 
Cluster-randomised trials: We will analyse cluster-randomised trials at the individual patient level, but we will account for the cluster design and seek statistical advice.

Studies with multiple treatment groups: In the pairwise meta-analyses we will treat studies with multiple treatment groups as different independent two-arm studies. In the network meta-analysis we will include all treatment arms from such studies and properly account for the correlation induced in the respective relative treatment effects.

Dealing with missing data-We will deal with missing data according to the recommendations in Chapter 16 of the Cochrane Handbook for Systematic Reviews of Interventions (Higgins 2011c). We will contact authors in order to obtain information that is missing or unclear in the published report. In trials that include thrombocytopenic participants with bone marrow failure as well as participants with other causes of thrombocytopenia, we will extract data for the bone marrow failure subgroup from the general trial data. If we cannot perform this step, we will contact the trial author. We will record the number of participants lost to follow-up for each trial. Where possible, we will analyse data according to the intention-to-treat (ITT) principle, but if insufficient data are available, we will present per protocol (PP) analyses (Higgins 2011c). We will perform a sensitivity analysis when possible to evaluate the robustness of results when we move away from the available case analysis using the informative missingness parameter framework. We will use this analysis to account for the uncertainty imposed in the analysis due to the presence of missing outcome data (Mavridis 2014; White 2008). We will perform this analysis using the metamiss2 command in Stata available from http://www.mtm.uoi.gr.

\section{Assessment of heterogeneity}

\section{Assessment of clinical and methodological heterogeneity within treatment}

comparisons: If we consider the clinical and methodological characteristics of individual studies to be sufficiently homogenous, we will combine the data to perform a meta-analysis (Deeks 2011).

If excessive heterogeneity is present, we will not report the overall summary statistic.

Assumptions when calculating the heterogeneity: In standard pairwise meta-analyses, we will estimate different heterogeneity variances for each pairwise comparison. In network meta-analysis we will assume a common estimate for the heterogeneity variance across the different comparisons.

Measures and tests for heterogeneity: We will assess statistical heterogeneity within each pairwise comparison using the $\mathrm{I}^{2}$ statistic and its $95 \%$ confidence interval, which measures the percentage of variability that cannot be attributed to random error $\left(\mathrm{I}^{2}>50 \%\right.$ moderate heterogeneity, $\mathrm{I}^{2}>80 \%$ considerable heterogeneity). The assessment of statistical heterogeneity in the entire network will be based on the magnitude of the heterogeneity variance parameter $\left(\mathrm{Tau}^{2}\right)$ estimated from the network meta-analysis models. We will compare the magnitude of the heterogeneity variance with previously suggested empirical distributions (Rhodes 2015; Turner 2012). We will also estimate a total $\mathrm{I}^{2}$ value for heterogeneity in the network and estimate prediction intervals for all relative effects 
(Jackson 2014; Riley 2011). We will explore potential causes of heterogeneity by subgroup and meta-regression analyses if possible (Deeks 2011).

Assessment of transitivity across treatment groups: We will assess the assumption of transitivity by comparing the distribution of the potential effect modifiers across the different pairwise comparisons. In this context we expect the transitivity assumption to hold, assuming that the baseline characteristics of patients in each study are similar with regard to severity of disease, baseline platelet count and co-interventions. We will epidemiologically evaluate the assumption of transitivity by comparing the clinical and methodological characteristics of sets of studies grouped by comparison (Jansen 2013; Salanti 2012).

Assessment of reporting biases-If sufficient studies are available, we will explore the presence of small-study effects in direct meta-analyses by generating a funnel plot and statistically using a linear regression test. We will consider a $P$ value of less than 0.10 significant for this test (Sterne 2011). We will also draw contour-enhanced funnel plots to assess whether publication bias is likely to operate (Peters 2008). We will use a comparisonadjusted funnel plot and network meta-regression models to assess the presence of smallstudy effects in the entire network (Chaimani 2012; Chaimani 2013).

\section{Data synthesis}

Methods for direct treatment comparisons: We will perform direct meta-analyses according to the recommendations in Chapter 9 of the Cochrane Handbook for Systematic Reviews of Interventions, using aggregated data for analyses (Deeks 2011). Where there is sufficient data with enough similarities (in participants, interventions, settings and outcome measurement) between the data, we will undertake meta-analyses using the Review Manager 5 software (RevMan 2014). One review author (MD) will enter the data into the software, and a second (AH) will then check for accuracy. Where meta-analysis is feasible, we will use the random-effects model to pool the data. We will use the Mantel-Haenszel method for dichotomous outcomes and the inverse variance method for continuous outcomes. We will employ the generic inverse variance method for time-to-event outcomes. We will use the fixed-effect model as a sensitivity analysis.

Methods for network meta-analysis: We will perform network meta-analysis in Stata using the method of multivariate meta-analysis that treats the different comparisons in studies as different outcomes (StataCorp 2011; White 2012). We will perform this analysis using a network package with the mvmeta command (White 2011; White 2015), and we will present the results using the network graphs package in Stata (Chaimani 2013; Chaimani 2015).

\section{Subgroup analysis and investigation of heterogeneity}

Assumptions when calculating the heterogeneity: In standard pairwise meta-analyses, we will estimate different heterogeneity variances for each pairwise comparison. In network meta-analysis, we will assume a common estimate for the heterogeneity variance across the different comparisons. 
Measures and tests for heterogeneity: We will assess statistical heterogeneity within each pairwise comparison using the $\mathrm{I}^{2}$ statistic and its $95 \%$ confidence interval, which measures the percentage of variability that cannot be attributed to random error. The assessment of statistical heterogeneity in the entire network will be based on the magnitude of the heterogeneity variance parameter $\left(\mathrm{Tau}^{2}\right)$ estimated from the network meta-analysis models. For dichotomous outcomes, we will compare the magnitude of the heterogeneity variance with the empirical distribution. We will also estimate a total $\mathrm{I}^{2}$ value for heterogeneity in the network.

\section{Investigation of heterogeneity and inconsistency}

Local approaches for evaluating inconsistency: To evaluate the presence of inconsistency locally, we will use the loop-specific approach. This method evaluates the consistency assumption in each closed loop of the network separately as the difference between direct and indirect estimates for a specific comparison in the loop inconsistency factor. The magnitude of the inconsistency factors and their $95 \%$ confidence intervals can be used to infer for the presence of inconsistency in each loop. We will assume a common heterogeneity estimate within each loop. We will present the results of this approach graphically in a forest plot using the ifplot command of the network graphs package in Stata (StataCorp 2011).

Global approaches for evaluating inconsistency: To evaluate the consistency assumption in the entire network simultaneously, we will use the design-by-treatment interaction model (Higgins 2012). This method accounts for two different sources of inconsistency that can occur when studies with different designs (for example two-arm trials compared to threearm trials) give different results as well as disagreement between direct and indirect evidence. Using this approach, we will infer for the presence of inconsistency from any source in the entire network based on a $\mathrm{Chi}^{2}$ test. We will perform the design-by-treatment model in Stata using the network package. Inconsistency and heterogeneity are interwoven; to distinguish between these two sources of variability, we will employ the $\mathrm{I}^{2}$ for inconsistency that measures the percentage of variability that cannot be attributed to random error or heterogeneity (Jackson 2014).

Subgroup analyses: If adequate data are available we will perform subgroup analyses and network meta-regression for each of the following variables in order to explain heterogeneity, inconsistency or both.

- $\quad$ Type of bone marrow failure disorder (myelodysplastic syndromes (MDS), aplastic anaemia, myelofibrosis or congenital bone marrow failure disorder).

- $\quad$ Severity of disease.

- $\quad$ Baseline platelet count.

- Study precision. 
Sensitivity analysis-We will assess the robustness of the overall results by performing the following sensitivity analyses where appropriate with respect to those trials deemed to be at high risk of bias. For dichotomous data, we will assess the influence of participant dropout, analysing separately RCTs with less than $20 \%$ drop-out, RCTs with $20 \%$ to $50 \%$ dropout and RCTs with greater than 50\% drop-out. We will also perform a sensitivity analysis to assess the robustness of results when we move away from the available case analysis (see Dealing with missing data). We will use the fixed-effect model for sensitivity analyses as part of the exploration of heterogeneity.

Summary of findings table: We will use an approach that extends the GRADE system into network meta-analysis to build a 'Summary of findings' table, as suggested in Chapter 11 of the Cochrane Handbook for Systematic Reviews of Interventions (Schünemann 2011;

Salanti 2014). The outcomes we will include for each type of comparison are listed below.

- $\quad$ Number of patients with at least one bleeding episode.

- $\quad$ Number of patients with life-threatening or fatal bleeding.

- $\quad$ Number of platelet transfusions per patient.

- $\quad$ Number of red cell transfusions per patient.

- $\quad$ Adverse events - thromboembolism.

- $\quad$ Adverse events - transfusion or drug reactions.

\section{ACKNOWLEDGEMENTS}

We thank the editorial base of the Cochrane Haematological Malignancies Review Group.

We thank the National Institute for Health Research (NIHR). This review is part of a series of reviews that have been partly funded by the NIHR Cochrane Programme Grant - Safe and Appropriate Use of Blood Components.

\section{SOURCES OF SUPPORT}

Internal sources

NHS Blood and Transplant, Research and Development, UK.

To fund the work of the Systematic Review Initiative (SRI)

External sources

- Cochrane Haematological Malignancies Group, Department for Internal Medicine, Germany. For editorial support

National Institute for Health Research (NIHR) Cochrane Programme Grant, UK.

To provide funding for systematic reviews and methodological support from the Centre for Statistics in Medicine, Oxford

\section{CONTRIBUTIONS OF AUTHORS}

Michael Desborough: protocol development, searching, selection of studies, eligibility and quality assessment, data extraction and analysis and

content expertise. 
Lise Estcourt: protocol development and content expertise.

Sally Hopewell: protocol development and methodological expertise.

Marialena Trivella: protocol development and statistical expertise.

Carolyn Doree: protocol development, searching and selection of studies.

Andreas Hadjinicolaou: protocol development, searching, selection of studies, eligibility and quality assessment, data extraction and analysis, and

content expertise.

Anna Chaimani: protocol development, statistical expert and network meta-analysis expertise.

Paresh Vyas: protocol development and content expertise.

Simon Stanworth: protocol development and content expertise.

\section{DECLARATIONS OF INTEREST}

Michael Desborough: none known.

Lise Estcourt: partly funded by the NIHR Cochrane Programme Grant - Safe and Appropriate Use of Blood Components.

Sally Hopewell: partly funded by the NIHR Cochrane Programme Grant - Safe and Appropriate Use of Blood Components.

Marialena Trivella: partly funded by the NIHR Cochrane Programme Grant - Safe and Appropriate Use of Blood Components.

Carolyn Doree: none known.

Andreas Hadjinicolaou: none known.

Anna Chaimani: none known.

Paresh Vyas: none known.

Simon Stanworth: none known.

\section{Appendix 1. CENTRAL search strategy}

\#1 MeSH descriptor: [Hematologic Neoplasms] explode all trees

\#2 MeSH descriptor: [Hematologic Diseases] this term only

\#3 MeSH descriptor: [Leukemia] explode all trees

\#4 MeSH descriptor: [Preleukemia] this term only 
\#5 MeSH descriptor: [Bone Marrow Diseases] explode all trees

\#6 MeSH descriptor: [Thrombocytopenia] explode all trees

\#7 MeSH descriptor: [Bone Marrow] this term only and with qualifier(s):

[Pathology - PA]

\#8 ((myelos* near/2 (nonleukemic or aleukemic)) or (myeloid near/2 metaplasia*) or myelofibros* or (bone marrow near/5 fibros*) or myeloscleros*)

\#9 (myelodysplas* or myeloid dysplasia or preleukemi* or preleukaemi* or dysmyelopoie* or 5Q syndrome)

\#10 ((aplast* or hypoplast* or refractory or aregenerative or sideroblastic or sideroachrestic or chronic*) near/2 an?emia)

\#11 ((haematolog* or hematolog* or hemato-oncolog* or haemato-oncolog*) near/2 patients)

\#12 ((haematolog* or hematolog* or blood or red cell* or white cell* or lymph* or marrow or platelet*) near/3 (malignan* or oncolog* or cancer* or neoplasm* or carcinoma*))

\#13 erythroid aplasia or erythrodysplas* or hematopoietic aplasia or pancytopen*

\#14 (IMF or PMF or MDS):ti

\#15 (bone marrow near/3 (fail* or disease* or disorder* or aplasia or dysplasia or hypoplasia))

\#16 (thrombocytop?eni* or leuk?emi* or myelodysplas* or myeloproliferat* or shwachman diamond or (dyskeratosis next congenita*) or AML)

\#17 (fanconi* next (an?emia or panmyelopathy or syndrome))

$\# 18 \# 1$ or \#2 or \#3 or \#4 or \#5 or \#6 or \#7 or \#8 or \#9 or \#10 or \#11 or \#12 or \#13 or \#14 or \#15 or \#16 or \#17

\#19 MeSH descriptor: [Factor VIIa] explode all trees

\#20 (factor viia or factor 7a or rfviia or fviia or novoseven* or novo seven* or eptacog* or proconvertin)

\#21 ((activated near/2 factor seven) or (activated near/2 factor vii) or (activated near/3 rfvii) or (activated near/2 fvii))

\#22 (factor seven or factor vii or factor 7):ti

\#23 \#19 or \#20 or \#21 or \#22

\#24 MeSH descriptor: [Fibrinogen] explode all trees

\#25 ("fibrinogen concentrate*" or "factor I" or haemocomplettan* or riastap*)

\#26 ((platelet* or thrombocyte*) near/5 (substitute* or artificial*))

\#27 platelet-poor plasma* 
\#29 MeSH descriptor: [Deamino Arginine Vasopressin] explode all trees

\#30 (desmopressin* or vasopressin deamino or nocutil or octim or minurin or deamino-8-d-arginine vasopressin or vasopressin 1-desamino-8-arginine or desmotabs or ddavp or ddavp or adiuretin or octostim or desmogalen)

\#31 MeSH descriptor: [Thrombopoietin] explode all trees and with qualifier(s): [Administration \& dosage - AD, Adverse effects - AE, Therapeutic use - TU]

\#32 MeSH descriptor: [Recombinant Fusion Proteins] explode all trees and with qualifier(s): [Administration \& dosage - AD, Adverse effects - AE, Therapeutic use - TU]

\#33 MeSH descriptor: [Receptors, Fc] explode all trees and with qualifier(s): [Administration \& dosage - AD, Therapeutic use - TU]

\#34 MeSH descriptor: [Receptors, Thrombopoietin] explode all trees and with qualifier(s): [Administration \& dosage - AD, Agonists - AG, Therapeutic use TU]

\#35

(eltrombopag* or promacta* or revolade* or romiplastin* or romiplostim* or nplate)

\#36 (amg531 or amg 531 or amg-531 or sb497115 or sb 497115 or sb-497115 or fab59 or fab 59 or fab-59 or AKR501 or AKR 501 or AKR-501 or YM477 or YM 477 or YM-477 or Peg-TPOmp*)

\#37 ((TPO or thrombopoietin) next (mimetic* or receptor agonist* or agonist* or agent*))

\#38 \#29 or \#30 or \#31 or \#32 or \#33 or \#34 or \#35 or \#36 or \#37

\#39 (((haemosta* or hemosta* or antihaemorrhag* or antihemorrhag* or anti haemorrhag* or anti-hemorrhag*) near/5 (drug* or agent* or treat* or therap*)) or ((coagulat* or clotting) adj factor*))

\#40 MeSH descriptor: [Interleukin-6] explode all trees

\#41 MeSH descriptor: [Interleukin-11] this term only

\#42 ("interleukin 6" or "interleukin 11" or IL6 or IL-6 or IL11 or IL-11 or sigosix or neumega or oprelvekin or rhIL-11 or INTLK11)

\#43 \#40 or \#41 or \#42

\#44 MeSH descriptor: [Factor XIII] explode all trees

\#45 factor xiii* or fxiii* or rfxiii* or stabili?ing factor fibrin or fibrin stabili?ing factor or laki-lorand factor or fibrinase or corifact or fibrogammin or tretten

\#46 \#44 or \#45

\#47 \#23 or \#28 or \#38 or \#39 or \#43 or \#46 
\#48

\#49

\#50

\#51

\#52

\#53

\#54

$\# 18$ and \#47

MeSH descriptor: [Antifibrinolytic Agents] this term only

MeSH descriptor: [Tranexamic Acid] this term only

MeSH descriptor: [Aminocaproic Acid] this term only

MeSH descriptor: [Aprotinin] this term only

antifibrinolytic* or anti-fibrinolytic* or antiplasmin* or "plasmin inhibitor*" or tranexamic or tranhexamic or "cyclohexanecarboxylic acid" or amcha or "trans-4-aminomethyl-cyclohexanecarboxylic acid" or "t-amcha" or amca or "kabi 2161" or transamin or exacyl or amchafibrin or anvitoff or spotof or cyklokapron or ugurol or amstat or antivoff or caprilon or aminomethylcyclohexanecarbonic or aminomethylcyclohexanecarboxylic or AMCHA or amchafibrin or amikapron or "aminomethyl cyclohexane carboxylic acid" or "aminomethyl cyclohexanecarboxylic acid" or "aminomethylcyclohexane carbonic acid" or "aminomethylcyclohexane carboxylic acid" or "aminomethylcyclohexanecarbonic acid" or "aminomethylcyclohexanecarboxylic acid" or "aminomethylcyclohexanocarboxylic acid" or "aminomethylcyclohexanoic acid" or "cl 65336" or cl65336 or cyclocapron or cyclokapron or cyklocapron or cyklokapron or exacyl or frenolyse or fibrinon or hemostan or hexacapron or hexakapron or kalnex or lysteda or rikaparin or ronex or theranex or tranexam or tranexanic or tranexic or "trans achma" or transexamic or trenaxin or TXA

Agretax or Bio-Stat or Capiloc or Capitrax or "Clip Inj” or Clot-XL or Clotawin-T or Coastat or Cuti or Cymin or Dubatran or Examic or Existat or Extam or Fibran or Gynae-Pil or Hemstate or Menogia or Monitex or Nestran or Nexamic or Nexi-500 or Nexmeff or Nixa-500 or Rheonex or "Sylstep TX" or Synostat or T-nex or T-Stat or Tanmic or Temsyl-T or Texakind or Texanis or Texapar or Texid or Thams or Tonopan or Traklot or Tramic or Tramix or Tranarest or "Trance Inj" or Tranecid or Tranee or Tranemic or Tranex or Tranexa or Tranfib or Tranlok or Transtat or Transys or Tranxi or Trapic or Traxage or Traxamic or Trenaxa or Trexamic or “Trim Inj” or Tx-1000 or Tx-500 or Wistran or X-Tran or Xamic

aminocaproic or aminohexanoic or amino-caproic or amino-n-hexanoic or cy-116 or cy 116 or lederle or acikaprin or afibrin or amicar or caprocid or capracid or capramol or caprogel or caprolest or caprolisin* or caprolysin* or capromol or epsikapron or hemocaprol or caproamin or EACA or caprolest or capralense or hexalense or hamostat or hemocid or "cl 10304" or cl10304 or ecapron or ekaprol or epsamon or epsicaprom or epsicapron or epsilcapramin or "epsilon amino caproate" or "epsilon aminocaproate" or epsilonaminocaproic or epsilonaminocapronsav or ethaaminocaproic or ethaaminocaproic or emocaprol or hepin or ipsilon or jd177or neocaprol or nsc26154 or resplamin or tachostyptan 
\#56 aprotinin* or antagosan or antilysin* or apronitin* or apronitrine or aprotimbin or aprotonin* or "bayer a 128 " or "bayer a128" or contrical or contrycal or contrykal or dilmintal or "frey inhibitor" or gordox or haemoprot or iniprol or kontrikal or kontrycal or "Kunitz inhibitor" or "Kunitz trypsin inhibitor" or midran or "pancreas antitrypsin" or "pancreatic antitrypsin" or protinin or pulmin or "riker 52g" or rivilina or "rp 9921" or rp9921 or tracylol or trascolan or trasilol or traskolan or trasylol or trazylol or "trypsin inhibitor" or zymofren or zymophren

$\# 57 \quad \# 49$ or $\# 50$ or $\# 51$ or $\# 52$ or \#53 or \#54 or \#55 or \#56

\#58 \#18 and \#57

\#59 \#48 or \#58

\section{Appendix 2. MEDLINE search strategy}

1. exp Hematologic Neoplasms/

2. Hematologic Diseases/

3. $\quad \operatorname{exp~Leukemia/~}$

4. Preleukemia/

5. $\quad \exp$ Bone Marrow Diseases/

6. Bone Marrow/pa

7. $\quad \exp$ Thrombocytopenia/

8. (bone marrow adj3 (fail* or disease* or disorder* or aplasia or hypoplasia or dysplasia)).tw,kf,ot.

9. (thrombocytop?eni* or thrombop?en* or leuk?emi* or myeloproliferat* or shwachman diamond or (dyskeratosis adj1 congenita*) or AML).tw,kf,ot.

10. (myelodysplas* or myeloid dysplasia or preleukemi* or preleukaemi* or dysmyelopoie* or 5Q syndrome).tw,kf,ot.

11. ((aplast* or hypoplast* or refractory or aregenerative or sideroblastic or sideroachrestic or chronic*) adj2 an?emia).tw,kf,ot.

12. (erythroid aplasia or erythrodysplas* or hematopoietic aplasia or pancytopen*).tw,kf,ot.

13. (fanconi* adj (an?emia or panmyelopathy or syndrome)).tw,kf,ot.

14. ((myelos* adj2 nonleukemic) or (myeloid adj2 metaplasia*) or myelofibros* or (bone marrow adj5 fibros*) or myeloscleros*).tw,kf,ot.

15. (IMF or PMF or MDS).ti.

16. ((haematolog* or hematolog* or blood or red cell* or white cell* or lymph* or marrow or platelet*) adj3 (malignan* or oncolog* or cancer* or neoplasm*)).tw,kf,ot. 
17. ((haematolog* or hematolog* or haemato-oncolog* or hemato-oncolog*) adj2 patients).tw,kf,ot.

$18 . \quad$ or/1-17

19. Factor VIIa/

20. (factor viia or factor 7a or rfviia or fviia or novoseven* or novo seven* or eptacog* or proconvertin).tw.

21. ((activated adj2 factor seven) or (activated adj2 factor vii) or (activated adj3 rfvii) or (activated adj2 fvii)).tw.

22. (factor seven or factor vii or factor 7).ti.

23. $\quad$ or $/ 19-22$

24. Fibrinogen/ad, ae, sd, tu, th

25. *Fibrinogen/

26. (fibrinogen concentrate* or factor I or haemocomplettan* or riastap*).tw.

27. $\quad$ or $/ 24-26$

28. ((platelet* or thrombocyte*) adj5 (substitute* or artificial*)).tw.

29. platelet-poor plasma*.tw.

30. $*$ Deamino Arginine Vasopressin/

31. Deamino Arginine Vasopressin/ad, ae, st, tu, to

32. (desmopressin* or vasopressin deamino or nocutil or octim or minurin or deamino-8-d-arginine vasopressin or vasopressin 1-desamino-8-arginine or desmotabs or DDAVP or adiuretin or octostim or desmogalen).tw.

33. or $/ 28-32$

34. Thrombopoietin/ad, tu

35. Recombinant Fusion Proteins/ad, tu

36. Receptors, Fc/ad, tu

37. Receptors, Thrombopoietin/ad, ag, ai, tu

38. (eltrombopag* or promacta* or revolade* or SB-497115-GR or romiplostim* or nplate* or AMG-31 or AMG31).tw.

39. (amg531 or amg 531 or amg-531 or sb497115 or sb 497115 or sb-497115 or fab59 or fab 59 or fab-59 or AKR501 or AKR 501 or AKR-501 or YM477 or YM 477 or YM-477 or Peg-TPOmp*).tw.

40. ((TPO or thrombopoietin) adj (mimetic* or receptor agonist* or agonist* or agent*)).tw.

41. $\quad$ or $/ 34-40$ 
42. (( (haemosta* or hemosta* or antihaemorrhag* or antihemorrhag* or anti haemorrhag* or anti-hemorrhag*) adj5 (drug* or agent* or treat* or therap*)) or ((coagulat* or clotting) adj factor*)).tw.

43. Interleukin-6/

44. ("interleukin 6" or IL6 or IL-6 or sigosix).tw.

45. Interleukin-11/

46. ("interleukin 11" or IL11 or IL-11 or neumega or oprelvekin or rhIL-11 or Intlk11).tw.

47. $\quad$ or $/ 43-46$

48. $\quad$ exp Factor XIII/

49. (factor xiii* or fxiii* or rfxiii* or stabili?ing factor fibrin or fibrin stabili? ing factor or laki lorand factor or fibrinase or corifact or fibrogammin or tretten).tw.

50. $\quad$ or $/ 48-49$

51. 23 or 27 or 33 or 41 or 42 or 47 or 50

52. randomized controlled trial.pt.

53. controlled clinical trial.pt.

54. randomi*.tw.

55. placebo.ab.

56. clinical trials as topic.sh.

57. randomly.ab.

58. groups.ab.

59. trial.ti.

60. $\quad$ or/52-59

61. Animals/ not Humans/

62. 60 not 61

63. Antifibrinolytic Agents/

64. Tranexamic Acid/

65. Aminocaproic Acid/

66. Aprotinin/

67. (antifibrinolytic* or anti-fibrinolytic* or antiplasmin* or plasmin inhibitor* or tranexamic or tranhexamic or cyclohexanecarboxylic acid* or amcha or trans-4-aminomethyl-cyclohexanecarboxylic acid* or t-amcha or amca or "kabi 2161" or transamin or exacyl or amchafibrin or anvitoff or 
spotof or cyklokapron or ugurol or aminomethylcyclohexanecarbonic acid or aminomethylcyclohexanecarboxylic acid or AMCHA or amchafibrin or amikapron or aminomethyl cyclohexane carboxylic acid or aminomethyl cyclohexanecarboxylic acid or aminomethylcyclohexane carbonic acid or aminomethylcyclohexane carboxylic acid or aminomethylcyclohexanecarbonic acid or aminomethylcyclohexanecarboxylic acid or aminomethylcyclohexanocarboxylic acid or aminomethylcyclohexanoic acid or amstat or antivoff or caprilon or cl?65336 or c165336 or cyclocapron or cyclokapron or cyklocapron or cyklokapron or exacyl or frenolyse or fibrinon or hemostan or hexacapron or hexakapron or kalnex or lysteda or rikaparin or ronex or theranex or tranexam or tranexanic or tranexic or trans achma or transexamic or trenaxin or TXA).tw.

68. (Agretax or Bio-Stat or Capiloc or Capitrax or Clip Inj or Clot-XL or Clotawin-T or Coastat or Cuti or Cymin or Dubatran or Examic or Existat or Extam or Fibran or Gynae-Pil or Hemstate or Menogia or Monitex or Nestran or Nexamic or Nexi-500 or Nexmeff or Nixa-500 or Rheonex or Sylstep TX or Synostat or T-nex or T Stat or T Stat or Tanmic or Temsyl-T or Texakind or Texanis or Texapar or Texid or Thams or Tonopan or Traklot or Tramic or Tramix or Tranarest or Trance Inj or Tranecid or Tranee or Tranemic or Tranex or Tranexa or Tranfib or Tranlok or Transtat or Transys or Tranxi or Trapic or Traxage or Traxamic or Trenaxa or Trexamic or Trim Inj or Tx-1000 or Tx 500 or Wistran or X-Tran or Xamic).tw.

69. (amino?caproic or amino?hexanoic or amino-caproic or amino-n-hexanoic or cy-116 or cy116 or lederle or acikaprin or afibrin or amicar or caprocid or capracid or capramol or caprogel or caprolest or caprolisin* or caprolysin* or capromol or epsikapron or hemocaprol or caproamin or EACA or caprolest or capralense or hexalense or hamostat or hemocid or cl 10304 or cl10304 or ecapron or ekaprol or epsamon or epsicaprom or epsicapron or epsilcapramin or epsilon amino caproate or epsilon aminocaproate or epsilonaminocaproic or epsilonaminocapronsav or etha? aminocaproic or ethaaminocaproich or emocaprol or hepin or ipsilon or jd?177or neocaprol or nsc? 26154 or resplamin or tachostyptan).tw.

70. (aprotinin or antagosan or antilysin* or apronitin* or apronitrine or aprotimbin or aprotinine or aprotonin or "bayer a 128 " or "bayer a128" or bovine pancreatic secretory trypsin inhibitor or contrical or contrycal or contrykal or dilmintal or frey inhibitor or gordox or haemoprot or iniprol or kallikrein trypsin inhibitor or kazal type trypsin inhibitor or kontrikal or kontrycal or Kunitz inhibitor or Kunitz trypsin inhibitor or midran or pancreas antitrypsin or pancreas secretory trypsin inhibitor or pancreas trypsin inhibitor or pancreatic antitrypsin or pancreatic secretory trypsin inhibitor or pancreatic trypsin inhibitor or protinin or pulmin or "riker 52g" or rivilina or "rp 9921" or rp9921 or tracylol or trascolan or trasilol 
or traskolan or trasylol or trazylol or trypsin inhibitor or zymofren or zymophren).tw.

71. or/63-70

72. 51 or 71

73. 18 and 72 and 62

\section{Appendix 3. EMBASE search strategy}

1.

2.

Hematologic Malignancy/

3.

exp Myeloproliferative Disorder/

4.

exp Aplastic Anemia/

5.

exp Thrombocytopenia/

(bone marrow adj3 (fail* or disease* or disorder* or aplasia or hypoplasia or dysplasia)).tw,kf,ot.

6. ((myelos* adj2 nonleukemic) or (myeloid adj2 metaplasia*) or myelofibros* or (bone marrow adj5 fibros*) or myeloscleros*).tw,kf,ot.

7. (thrombocytop?eni* or thrombop?en* oro leuk?emi* or myeloproliferat* or shwachman diamond or (dyskeratosis adj1 congenita*) or AML).tw,kf,ot.

8. (erythroid aplasia or erythrodysplas* or hematopoietic aplasia or pancytopen*).tw,kf,ot.

9. (fanconi* adj (an?emia or panmyelopathy or syndrome)).tw.

10. ((haematolog* or hematolog* or haemato-oncolog* or hemato-oncolog*) $\operatorname{adj} 2$ patients).tw,kf,ot.

11. ((haematolog* or hematolog* or blood or red cell* or white cell* or lymph* or marrow or platelet*) adj3 (malignan* or oncolog* or cancer* or neoplasm*)).tw,kf,ot.

12. $\quad$ exp Myelodysplastic Syndrome/

13. Myelodysplasia/ or Preleukemia/

14. (myelodysplas* or myeloid dysplasia or preleukemi* or preleukaemi* or dysmyelopoie* or 5Q syndrome).tw,kf,ot.

15. ((aplast* or hypoplast* or refractory or aregenerative or sideroblastic or sideroachrestic or chronic*) adj2 an?emia).tw,kf,ot.

16. (MDS or IMF or PMF).ti.

17. $\quad$ or/1-16

18. Factor VIIa/ 
19. (factor viia or factor $7 \mathrm{a}$ or rfviia or fviia or novoseven* or novo seven* or eptacog* or proconvertin).tw.

20. ((activated adj2 factor seven) or (activated adj2 factor vii) or (activated adj3 rfvii) or (activated adj2 fvii)).tw.

21. (factor seven or factor vii or factor 7).ti.

22. $\quad$ or $/ 18-21$

23. Fibrinogen/ae, ct, ad, cb, cm, cr, dv, do, dt, to, iv, pa, sc, th

24. Fibrinogen Concentrate/

25. (fibrinogen concentrate* or factor I or haemocomplettan* or riastap*).tw.

26. $\quad$ or $/ 23-25$

27. ((platelet* or thrombocyte*) adj5 (substitute* or artificial*)).tw.

28. platelet-poor plasma*.tw.

29. *Desmopressin/

30. Desmopressin/ad, ae, dt

31. (desmopressin* or vasopressin deamino or nocutil or octim or minurin or deamino-8-d-arginine vasopressin or vasopressin 1-desamino-8-arginine or desmotabs or ddavp or ddavp or adiuretin or octostim or desmogalen).tw.

32. $\quad$ or $/ 27-31$

33. $\quad$ *Thrombopoietin Receptor/

34. Eltrombopag/

35. Romiplostim/

36. (eltrombopag* or promacta* or revolade* or SB-497115-GR or romiplostim* or nplate* or AMG-31 or AMG31).tw.

37. (amg531 or amg 531 or amg-531 or sb497115 or sb 497115 or sb-497115 or fab59 or fab 59 or fab-59 or AKR501 or AKR 501 or AKR-501 or YM477 or YM 477 or YM-477 or Peg-TPOmp*).tw.

38. ((TPO or thrombopoietin) adj (mimetic* or receptor agonist* or agonist*)).tw.

39. $\quad$ or $/ 33-38$

40. (( (haemosta* or hemosta* or antihaemorrhag* or antihemorrhag* or anti haemorrhag* or anti-hemorrhag*) adj5 (drug* or agent* or treat* or therap*)) or ((coagulat* or clotting) adj factor*)).tw.

41. blood clotting factor 13 / or blood clotting factor 13 concentrate/ or blood clotting factor $13 \mathrm{a} /$ or blood clotting factor $13 \mathrm{~b} /$ 
42. (factor xiii* or fxiii or rfxiii or stabili?ing factor fibrin or fibrin stabili?ing factor or laki lorand factor or fibrinase or corifact or fibrogammin or tretten).tw.

43. $\quad$ or/41-42

44. $\quad *$ interleukin $6 /$

45. interleukin 6/ae, ct, ad, an, cb, cm, cr, dv, do, it, dt, to, ei, ih, ar, ce, cv, ci, dl, ig, ly, im, na, ip, pl, sp, tl, tr, tu, iv, vi, po, pr, pe, pk, pd, rc, cj, sc, tp

46. ("interleukin 6" or IL6 or IL-6).ti.

47. interleukin $11 /$

48. ("interleukin 11" or IL11 or IL-11 or sigosix or neumega or oprelvekin or rhIL-11 or INTLK11).tw.

49. $\quad$ or/44-48

50. 22 or 26 or 32 or 39 or 40 or 43 or 49

51. Randomized Controlled Trial/

52. Randomization/

53. Single Blind Procedure/

54. Double Blind Procedure/

55. Crossover Procedure/

56. Placebo/

57. $\quad \exp$ Clinical Trial/

58. Prospective Study/

59. (randomi* or double-blind* or single-blind* or RCT*).tw.

60. (random* adj2 (allocat* or assign* or divid* or receiv*)).tw.

61. (crossover* or cross over* or cross-over* or placebo*).tw.

62. ((treble or triple) adj blind*).tw.

63. or/51-62

64. Case Study/

65. case report*.tw.

66. (note or editorial).pt.

67. $\quad$ or/64-66

68. $63 \operatorname{not} 67$

69. $\quad \exp$ Antifibrinolytic Agents/ 
70. (antifibrinolytic* or anti-fibrinolytic* or antiplasmin* or plasmin inhibitor* or tranexamic or tranhexamic or cyclohexanecarboxylic acid* or amcha or trans-4-aminomethyl-cyclohexanecarboxylic acid* or t-amcha or amca or "kabi 2161" or transamin or exacyl or amchafibrin or anvitoff or spotof or cyklokapron or ugurol or aminomethylcyclohexanecarbonic acid or aminomethylcyclohexanecarboxylic acid or AMCHA or amchafibrin or amikapron or aminomethyl cyclohexane carboxylic acid or aminomethyl cyclohexanecarboxylic acid or aminomethylcyclohexane carbonic acid or aminomethylcyclohexane carboxylic acid or aminomethylcyclohexanecarbonic acid or aminomethylcyclohexanecarboxylic acid or aminomethylcyclohexanocarboxylic acid or aminomethylcyclohexanoic acid or amstat or antivoff or caprilon or cl?65336 or cl65336 or cyclocapron or cyclokapron or cyklocapron or cyklokapron or exacyl or frenolyse or fibrinon or hemostan or hexacapron or hexakapron or kalnex or lysteda or rikaparin or ronex or theranex or tranexam or tranexanic or tranexic or trans achma or transexamic or trenaxin or TXA).tw.

71. (Agretax or Bio-Stat or Capiloc or Capitrax or Clip Inj or Clot-XL or Clotawin-T or Coastat or Cuti or Cymin or Dubatran or Examic or Existat or Extam or Fibran or Gynae-Pil or Hemstate or Menogia or Monitex or Nestran or Nexamic or Nexi-500 or Nexmeff or Nixa-500 or Rheonex or Sylstep TX or Synostat or T-nex or T Stat or T Stat or Tanmic or Temsyl-T or Texakind or Texanis or Texapar or Texid or Thams or Tonopan or Traklot or Tramic or Tramix or Tranarest or Trance Inj or Tranecid or Tranee or Tranemic or Tranex or Tranexa or Tranfib or Tranlok or Transtat or Transys or Tranxi or Trapic or Traxage or Traxamic or Trenaxa or Trexamic or Trim Inj or Tx-1000 or Tx 500 or Wistran or X-Tran or Xamic).tw.

72. (amino?caproic or amino?hexanoic or amino-caproic or amino-n-hexanoic or cy-116 or cy116 or lederle or acikaprin or afibrin or amicar or caprocid or capracid or capramol or caprogel or caprolest or caprolisin* or caprolysin* or capromol or epsikapron or hemocaprol or caproamin or EACA or caprolest or capralense or hexalense or hamostat or hemocid or cl 10304 or cl10304 or ecapron or ekaprol or epsamon or epsicaprom or epsicapron or epsilcapramin or epsilon amino caproate or epsilon aminocaproate or epsilonaminocaproic or epsilonaminocapronsav or etha? aminocaproic or ethaaminocaproich or emocaprol or hepin or ipsilon or jd?177or neocaprol or nsc? 26154 or resplamin or tachostyptan).tw.

73. (aprotinin or antagosan or antilysin* or apronitin* or apronitrine or aprotimbin or aprotinine or aprotonin or "bayer a 128" or "bayer a128" or bovine pancreatic secretory trypsin inhibitor or contrical or contrycal or contrykal or dilmintal or frey inhibitor or gordox or haemoprot or iniprol or kallikrein trypsin inhibitor or kazal type trypsin inhibitor or kontrikal or kontrycal or Kunitz inhibitor or Kunitz trypsin inhibitor or midran or 
pancreas antitrypsin or pancreas secretory trypsin inhibitor or pancreas trypsin inhibitor or pancreatic antitrypsin or pancreatic secretory trypsin inhibitor or pancreatic trypsin inhibitor or protinin or pulmin or "riker $52 \mathrm{~g}$ " or rivilina or "rp 9921" or rp9921 or tracylol or trascolan or trasilol or traskolan or trasylol or trazylol or trypsin inhibitor or zymofren or zymophren).tw.

74. $\quad$ or $/ 69-73$

75. 50 or 74

76. $\quad 17$ and 75 and 68

77. limit 76 to embase

\section{Appendix 4. CINAHL search strategy}

S1 (MH "Hematologic Neoplasms+")

S2 (MH Leukemia+)

S3 (MH “Anemia, Aplastic+")

S4 (MH "Bone Marrow Diseases+")

S5 (MH Thrombocytopenia+)

S6 (thrombocytopeni* or thrombocytopaeni* or thrombopeni* or thrombopaeni* or leukemi* or leukaemi* or myelodysplas* or myeloproliferat* or myelofibros* or AML or shwachman diamond or (dyskeratosis N1 congenita*))

S7 (myelodysplas* or bone marrow dysplas* or preleukemi* or preleukaemi* or dysmyelopoie* or 5Q syndrome)

S8 ((aplast* or hypoplast* or refractory or aregenerative or sideroblastic or sideroachrestic or chronic *) N2 (anemia or anaemia))

S9 ((myelos* $\mathrm{N} 2$ (nonleukemic or aleukemic)) or (myeloid N2 metaplasia*) or myelofibros* or (bone marrow N5 fibros*) or myeloscleros*)

S10 MDS or PMF or IMF or pancytopen* or erythroid aplasia or erythrodysplas* or hematopoietic aplasia

S11 (bone marrow N3 (fail* or disease* or disorder* or aplasia or dysplasia or hypoplasia))

S12 (fanconi* N2 (anemia or anaemia or panmyelopathy or syndrome))

S13 ((haematolog* or hematolog* or blood or red cell* or white cell* or lymph* or marrow or platelet*) N3 (malignan* or oncolog* or cancer* or neoplasm*))

S14 ((haematolog* or hematolog* or haemato-oncolog* or hemato-oncolog*) N2 patients) 
S15 S1 OR S2 OR S3 OR S4 OR S5 OR S6 OR S7 OR S8 OR S9 OR S10 OR S11 OR S12 OR S13 OR S14

S16 (MH "Blood Coagulation Factors+")

S17 (factor viia or factor 7a or rfviia or fviia or novoseven* or novo seven* or eptacog* or proconvertin* or fibrinogen concentrate* or factor I or haemocomplettan* or riastap*)

S18 ((activated N2 factor seven) or (activated N2 factor vii) or (activated N3 rfvii) or (activated $\mathrm{N} 2 \mathrm{fvii})$ )

S19 TI (factor seven or factor vii or factor 7)

S20 S16 OR S17 OR S18 OR S19

S21 ((platelet* or thrombocyte*) N5 (substitute* or artificial*))

S22 platelet-poor plasma*

S23 (MH "Desmopressin")

S24 (desmopressin* or vasopressin deamino or nocutil or octim or minurin or deamino-8-d-arginine vasopressin or vasopressin 1-desamino-8-arginine or desmotabs or ddavp or ddavp or adiuretin or octostim or desmogalen)

S25 S21 OR S22 OR S23 OR S24

S26 (MH "Benzoic Acids Therapeutic Use")

S27 (MH "Receptors, Cell Surface Therapeutic Use")

S28 (eltrombopag* or promacta* or revolade* or romiplastin* or romiplostim* or nplate or TPO*)

S29 (amg531 or amg 531 or amg-531 or sb497115 or sb 497115 or sb-497115 or fab59 or fab 59 or fab-59 or AKR501 or AKR 501 or AKR-501 or YM477 or YM 477 or YM-477 or Peg-TPOmp*)

S30 ((TPO or thrombopoietin) W1 (mimetic* or receptor agonist* or agonist* or agent*))

S31 S26 OR S27 OR S28 OR S29 OR S30

S32 (((haemosta* or hemosta* or antihaemorrhag* or antihemorrhag* or anti haemorrhag* or anti-hemorrhag*) N5 (drug* or agent* or treat* or therap*)) or ((coagulat* or clotting) W1 factor*))

S33 S20 OR S25 OR S31 OR S32

S34 S15 AND S33

S35 (MH Clinical Trials+)

S36 PT Clinical Trial

S37 TI ((controlled trial*) or (clinical trial*)) OR AB ((controlled trial*) or (clinical trial*)) 
S38 TI ((singl* blind*) OR (doubl* blind*) OR (trebl* blind*) OR (tripl* blind*) OR (singl* mask*) OR (doubl* mask*) OR (tripl* mask*)) OR AB ((singl* blind*) OR (doubl* blind*) OR (trebl* blind*) OR (tripl* blind*) OR (singl* mask*) OR (doubl* mask*) OR (tripl* mask*))

S39 TI randomi* OR AB randomi*

S40 MH RANDOM ASSIGNMENT

S41 TI ((phase three) or (phase III) or (phase three)) or AB ((phase three) or (phase III) or (phase three))

S42 (TI (random* N2 (assign* or allocat*))) OR (AB (random* N2 (assign* or allocat*)))

S43 MH PLACEBOS

S44 MH META ANALYSIS

S45 MH SYSTEMATIC REVIEW

S46 TI ("meta analys*" OR metaanalys* OR "systematic review" OR "systematic overview" OR "systematic search*”) OR AB ("meta analys*" OR metaanalys* OR "systematic review" OR "systematic overview" OR "systematic search*”)

S47 TI ("literature review" OR "literature overview" OR "literature search*”) OR AB ("literature review" OR "literature overview" OR "literature search*”)

S48 TI (cochrane OR embase OR cinahl OR cinhal OR lilacs OR BIDS OR science AND citation AND index OR cancerlit) OR AB (cochrane OR embase OR cinahl OR cinhal OR lilacs OR BIDS OR science AND citation AND index OR cancerlit)

S49 TI placebo* OR AB placebo*

S50 MH QUANTITATIVE STUDIES

S51 S35 or S36 or S37 or S38 or S39 or S40 or S41 or S42 or S43 or S44 or S45 or $\mathrm{S} 46$ or S47 or S48 or S49 or S50

S52 S34 AND S51

S53 (MM “Interleukins”)

S54 "interleukin 6" or "interleukin 11" or IL6 or IL-6 or IL11 or IL-11 or sigosix or neumega or oprelvekin or rhIL-11 or INTLK11

S55 factor xiii* or fxiii* or rfxiii* or stabili?ing factor fibrin or fibrin stabili?ing factor or laki-lorand factor or fibrinase or corifact or fibrogammin or tretten

S56 S53 OR S54 OR S55

S57 S33 OR S56

S58 S15 AND S51 AND S57

S59 (MH “Antifibrinolytic Agents”) 
S60

S61

S62

S63

S64

\section{S65}

S66

S67

S68

S69

(MH “Aminocaproic Acids”)

(MH “Tranexamic Acid”)

(MH “Aprotinin”)

TI ((antifibrinolytic* or anti-fibrinolytic* or antiplasmin* or "plasmin inhibitor*" or tranexamic or tranhexamic or "cyclohexanecarboxylic acid" or amcha or "trans-4-aminomethyl-cyclohexanecarboxylic acid" or "t-amcha" or amca or "kabi 2161" or transamin or exacyl or amchafibrin or anvitoff or spotof or cyklokapron or ugurol or amstat or antivoff or caprilon or aminomethylcyclohexanecarbonic or aminomethylcyclohexanecarboxylic or AMCHA or amchafibrin or amikapron or "aminomethyl cyclohexane ca ...

TI ((Agretax or Bio-Stat or Capiloc or Capitrax or "Clip Inj““ or Clot-XL or Clotawin-T or Coastat or Cuti or Cymin or Dubatran or Examic or Existat or Extam or Fibran or Gynae-Pil or Hemstate or Menogia or Monitex or Nestran or Nexamic or Nexi-500 or Nexmeff or Nixa-500 or Rheonex or "Sylstep TX“ or Synostat or T-nex or T-Stat or Tanmic or Temsyl-T or Texakind or Texanis or Texapar or Texid or Thams or Tonopan or Traklot or Tramic or Tramix or Tranarest or "Trance Inj“" or Tranecid or Tranee or ...

TI ((aminocaproic or aminohexanoic or amino-caproic or amino-n-hexanoic or cy-116 or cy 116 or lederle or acikaprin or afibrin or amicar or caprocid or capracid or capramol or caprogel or caprolest or caprolisin* or caprolysin* or capromol or epsikapron or hemocaprol or caproamin or EACA or caprolest or capralense or hexalense or hamostat or hemocid or "cl 10304" or cl10304 or ecapron or ekaprol or epsamon or epsicaprom or epsicapron or epsilcapramin or "epsilon amino caproate" or "epsilon amino ...

66 TI ((aprotinin* or antagosan or antilysin* or apronitin* or apronitrine or aprotimbin or aprotonin* or "bayer a 128 " or "bayer a128" or contrical or contrycal or contrykal or dilmintal or "frey inhibitor" or gordox or haemoprot or iniprol or kontrikal or kontrycal or "Kunitz inhibitor" or "Kunitz trypsin inhibitor" or midran or "pancreas antitrypsin" or "pancreatic antitrypsin" or protinin or pulmin or "riker 52g" or rivilina or "rp 9921" or rp9921 or tracylol or trascolan or trasilol or trasko ...

\section{S59 OR S60 OR S61 OR S62 OR S63 OR S64 OR S65 OR S66}

$$
\text { S57 OR S67 }
$$

S15 AND S51 AND S68

\section{Appendix 5. PubMed epublications search strategy}

\#1 (bone marrow failure OR bone marrow disease* or bone marrow disorder* OR bone marrow aplasia OR bone marrow dysplasia OR bone marrow hypoplasia OR aplastic anemia OR aplastic anaemia OR hypoplastic anemia OR hypoplastic anaemia OR refractory anemia OR refractory anaemia OR 
sideroblastic anemia OR sideroblastic anaemia OR a regenerative anemia OR aregenerative anaemia OR chronic anemia OR chronic anaemia OR fanconi OR erythroid aplasia OR erythrodysplas* OR hematopoietic aplasia OR haematopoietic aplasia OR pancytopen*)

\#2 (thrombocytop* OR leukemi* OR leukaemi* OR preleuk* OR myelodysplas* OR myeloproliferat* OR myelofibros* OR myeloscleros* OR shwachman diamond OR dyskeratosis congenital OR AML OR dysmyelopoie* or 5Q syndrome)

\#3 ((haematolog* OR hematolog* OR blood OR red cell* OR white cell* OR marrow OR platelet*) AND (malignan* OR oncolog* OR cancer OR cancers OR neoplasm* or carcinoma*))

\#4 ((myelos* AND (nonleukemic OR aleukemic)) OR (myeloid AND metaplasia*) OR (bone marrow AND fibros*))

\#5 IMF[TI] OR PMF[TI] OR MDS[TI]

\#6 (haematolog* patients OR hematolog* patients OR haemato-oncolog* patients OR hemato-oncolog* patients)

\#7 \#1 OR \#2 OR \#3 OR \#4 OR \#5 OR \#6

\#8 ("factor viia"[TI] OR "factor 7a"[TI] OR rfviia[TI] OR fviia[TI] OR novoseven*[TI] OR “novo seven*”[TI] OR eptacog*[TI] OR proconvertin[TI] OR “fibrinogen concentrate*”[TI] OR "factor I"[TI] OR haemocomplettan[TI] OR octafibrin[TI] OR riastap[TI])

\#9 "activated factor seven"[TI] OR "activated factor vii"[TI] OR "activated rfvii" [TI] OR "activated fvii”[TI] OR "factor seven"[TI] OR "factor vii”"[TI] OR "factor 7"[TI]

\#10 ((platelet* OR thrombocyte*) AND (substitute* OR artificial*))

\#11 ("platelet-poor plasma" OR desmopressin* OR vasopressin deamino OR nocutil OR octim OR minurin OR deamino-8-d-arginine vasopressin OR vasopressin 1-desamino-8-arginine OR desmotabs OR ddavp or adiuretin OR octostim OR desmogalen)

\#12 (eltrombopag* OR promacta* OR revolade* OR romiplastin* OR romiplostim* OR nplate*)

\#14 ("interleukin 6" or "interleukin 11" or IL6 or IL-6 or IL11 or IL-11 OR sigosix OR neumega OR oprelvekin OR rhIL-11 or INTLK11)

\#15 (factor xiii* OR fxiii* OR rfxiii* OR stabilizing factor fibrin OR stabilising factor fibrin OR fibrin stabilizing factor OR fibrin stabilising factor OR laki lorand factor OR fibrinase OR corifact OR fibrogammin OR tretten)

\#16 (amg531 OR amg 531 OR amg-531 OR sb497115 OR sb 497115 OR sb-497115 OR fab59 OR fab 59 OR fab-59 OR AKR501 OR AKR 501 OR AKR-501 OR YM477 OR YM 477 OR YM-477 OR Peg-TPOmp*) 
\#17 ((TPO OR thrombopoietin) AND (mimetic* OR receptor agonist* OR agonist* OR agent*))

\#18 ((haemosta* OR hemosta* OR antihaemorrhag* OR antihemorrhag* OR anti haemorrhag* OR anti-hemorrhag*) AND (drug OR drugs OR agent* OR treatment* OR therapy OR therapies)) OR (coagulat* factor OR clotting factor OR coagulat* factors OR clotting factors))

(antifibrinolytic[TI] OR anti-fibrinolytic[TI] OR antifibrinolytics[TI] OR antifibrinolytics[TI] OR antiplasmin[TI] OR antiplasmins[TI] OR "plasmin inhibitor*”[TI] OR tranexamic[TI] OR tranhexamic[TI] OR "cyclohexanecarboxylic acid"[TI] OR amcha[TI] OR "trans-4-aminomethylcyclohexanecarboxylic acid"[TI] OR "t-amcha"[TI] OR amca[TI] OR "kabi 2161"[TI] OR transamin[TI] OR exacyl[TI] OR amchafibrin[TI] OR anvitoff[TI] OR spotof[TI] OR cyklokapron[TI] OR ugurol[TI] OR amstat[TI] OR antivoff[TI] OR caprilon[TI] OR aminomethylcyclohexanecarbonic[TI] OR aminomethylcyclohexanecarboxylic[TI] OR AMCHA[TI] OR amchafibrin[TI] OR amikapron[TI] OR "aminomethyl cyclohexane carboxylic acid"[TI] OR "aminomethyl cyclohexanecarboxylic acid"[TI] OR "aminomethylcyclohexane carbonic acid"[TI] OR "aminomethylcyclohexane carboxylic acid"[TI] OR "aminomethylcyclohexanecarbonic acid"[TI] OR "aminomethylcyclohexanecarboxylic acid"[TI] OR "aminomethylcyclohexanocarboxylic acid"[TI] OR “aminomethylcyclohexanoic acid”[TI] OR “cl 65336"[TI] OR cl65336[TI] OR cyclocapron[TI] OR cyclokapron[TI] OR cyklocapron[TI] OR cyklokapron[TI] OR exacyl[TI] OR frenolyse[TI] OR fibrinon[TI] OR hemostan[TI] OR hexacapron[TI] OR hexakapron[TI] OR kalnex[TI] OR lysteda[TI] OR rikaparin[TI] OR ronex[TI] OR theranex[TI] OR tranexam[TI] OR tranexanic[TI] OR tranexic[TI] OR "trans achma"[TI] OR transexamic[TI] OR trenaxin[TI] OR TXA[TI] OR Agretax[TI] OR Bio-Stat[TI] OR Capiloc[TI] OR Capitrax[TI] OR “Clip Inj”[TI] OR Clot-XL[TI] OR Clotawin-T[TI] OR Coastat[TI] OR Cuti[TI] OR Cymin[TI] OR Dubatran[TI] OR Examic[TI] OR Existat[TI] OR Extam[TI] OR Fibran[TI] OR GynaePil[TI] OR Hemstate[TI] OR Menogia[TI] OR Monitex[TI] OR Nestran[TI] OR Nexamic[TI] OR Nexi-500[TI] OR Nexmeff[TI] OR Nixa-500[TI] OR Rheonex[TI] OR “Sylstep TX”[TI] OR Synostat[TI] OR T-nex[TI] OR TStat[TI] OR Tanmic[TI] OR Temsyl-T[TI] OR Texakind[TI] OR Texanis[TI] OR Texapar[TI] OR Texid[TI] OR Thams[TI] OR Tonopan[TI] OR Traklot[TI] OR Tramic[TI] OR Tramix[TI] OR Tranarest[TI] OR “Trance Inj”[TI] OR Tranecid[TI] OR Tranee[TI] OR Tranemic[TI] OR Tranex[TI] OR Tranexa[TI] OR Tranfib[TI] OR Tranlok[TI] OR Transtat[TI] OR Transys[TI] OR Tranxi[TI] OR Trapic[TI] OR Traxage[TI] OR Traxamic[TI] OR Trenaxa[TI] OR Trexamic[TI] OR “Trim Inj”[TI] OR Tx-1000[TI] OR Tx-500[TI] OR Wistran[TI] OR X-Tran[TI] OR Xamic [TI] OR aminocaproic[TI] OR aminohexanoic[TI] OR amino-caproic[TI] OR amino-n-hexanoic[TI] OR cy-116[TI] OR cy116[TI] OR lederle[TI] OR acikaprin[TI] OR afibrin[TI] OR 
amicar[TI] OR caprocid[TI] OR capracid[TI] OR capramol[TI] OR caprogel[TI] OR caprolest[TI] OR caprolisin[TI] OR caprolysin[TI] OR capromol[TI] OR epsikapron[TI] OR hemocaprol[TI] OR caproamin[TI] OR EACA[TI] OR caprolest[TI] OR capralense[TI] OR hexalense[TI] OR hamostat[TI] OR hemocid[TI] OR “cl 10304" [TI] OR cl10304[TI] OR ecapron[TI] OR ekaprol[TI] OR epsamon[TI] OR epsicaprom[TI] OR epsicapron[TI] OR epsilcapramin[TI] OR "epsilon amino caproate"[TI] OR "epsilon aminocaproate"[TI] OR epsilonaminocaproic[TI] OR epsilonaminocapronsav[TI] OR ethaaminocaproic[TI] OR ethaaminocaproic[TI] OR emocaprol[TI] OR hepin[TI] OR ipsilon[TI] OR jd177or neocaprol[TI] OR nsc26154[TI] OR resplamin[TI] OR tachostyptan[TI] OR aprotinin*[TI] OR antagosan[TI] OR antilysin*[TI] OR apronitin*[TI] OR apronitrine[TI] OR aprotimbin[TI] OR aprotonin*[TI] OR "bayer a 128"[TI] OR "bayer a128"[TI] OR contrical[TI] OR contrycal[TI] OR contrykal[TI] OR dilmintal[TI] OR "frey inhibitor" [TI] OR gordox[TI] OR haemoprot[TI] OR iniprol[TI] OR kontrikal[TI] OR kontrycal[TI] OR "Kunitz inhibitor" [TI] OR "Kunitz trypsin inhibitor"[TI] OR midran[TI] OR "pancreas antitrypsin"[TI] OR "pancreatic antitrypsin"[TI] OR protinin[TI] OR pulmin[TI] OR “riker 52g”[TI] OR rivilina[TI] OR “rp 9921”[TI] OR rp9921[TI] OR tracylol[TI] OR trascolan[TI] OR trasilol[TI] OR traskolan[TI] OR trasylol[TI] OR trazylol[TI] OR "trypsin inhibitor"[TI] OR zymofren[TI] OR zymophren[TI])

\#8 OR \#9 OR \#10 OR \#11 OR \#12 OR \#13 OR \#14 OR \#15 OR \#16 OR \#17 OR \#18 OR \#19

\#21 \#7 AND \#20

\#22 (random* OR blind* OR control group* OR placebo OR controlled trial OR controlled study OR trials OR systematic review OR meta-analysis OR metaanalysis OR literature OR medline OR cochrane OR embase) AND ((publisher[sb] OR inprocess[sb]) NOT pubstatusnihms)

\#23 \#21 AND \#22

\section{Appendix 6. Transfusion Evidence Library search strategy}

All Fields: (haematological OR hematological OR haematology OR hematology OR haemato-oncology OR hemato-oncology OR bone marrow failure OR bone marrow disease OR bone marrow disorder OR leukemia OR leukaemia OR preleukemia OR preleukaemia OR aplastic OR hypoplastic OR refractory OR sideroblastic OR fanconi OR thrombocytopenia OR thrombocytopenic OR myelodysplasia OR bone marrow dysplasia OR myeloproliferative OR myelofibrosis OR fibrosis OR myelosclerosis OR shwachman OR dyskeratosis OR AML OR dysmyelopoiesis OR 5Q syndrome) AND (antifibrinolytics OR factor viia OR fibrinogen OR haemocomplettan OR platelet-poor plasma OR eltrombopag OR promacta OR revolade OR romiplastim OR romiplostim OR nplate OR thrombopoietin receptor agonist OR thrombopoietin agonist OR TPO OR interleukin OR sigosix OR neumega OR oprelvekin OR factor xiii OR factor xiiia OR fibrinase OR fxiii OR 
rfxiii OR fxiiia OR rfxiiia OR corifact OR fibrogammin OR tretten OR tranexamic OR aprotinin OR EACA OR aminocaproic)

\title{
Appendix 7. LILACS search strategy
}

tw:(((haematological OR hematological OR haematology OR hematology OR haematooncology OR hemato-oncology OR bone marrow failure OR bone marrow disease OR bone marrow disorder OR leukemia OR leukemia OR preleukemia OR preleukemia OR aplastic OR hypoplastic OR refractory OR sideroblastic OR fanconi OR thrombocytopenia OR thrombocytopenic OR myelodysplasia OR bone marrow dysplasia OR myeloproliferative OR myelofibrosis OR fibrosis OR myelosclerosis OR shwachman OR dyskeratosis OR aml OR dysmyelopoiesis OR 5q syndrome) AND (factor viia OR factor 7a OR rfviia OR fviia OR novoseven OR novo seven OR eptacog OR proconvertin OR fibrinogen concentrate OR factor i OR haemocomplettan OR octafibrin OR riastap OR activated factor seven OR activated factor vii OR activated rfvii OR activated fvii OR factor seven OR factor vii OR factor 7 OR platelet-poor plasma OR desmopressin OR eltrombopag OR promacta OR revolade OR romiplastin OR romiplostim OR nplate OR thrombopoietin receptor OR thrombopoietin agonist OR thrombopoietin mimetic OR agonist OR TPO OR interleukin OR sigosix OR neumega OR oprelvekin OR factor xiii OR FXIII OR FXIIIa OR rFXIII OR rFXIIIa OR corifact OR fibrogammin OR tretten OR tranexamic OR aprotinin OR EACA OR aminocaproic) AND type of 'study:(clinical'trials OR systematic 'reviews))) AND (instance:"regional") AND db:(LILACS)

\section{Appendix 8. INDMED search strategy}

((factor viia OR rfvii OR rfviia OR fvii OR fviia OR factor seven OR factor vii OR novoseven OR novo seven OR eptacog OR proconvertin OR fibrinogen OR factor I OR haemocomplettan OR octafibrin OR riastap OR platelet-poor plasma OR desmopressin OR eltrombopag OR promacta OR revolade OR romiplastin OR romiplostim OR nplate OR thrombopoietin OR interleukin OR IL-6 OR IL-11 OR sigosix OR neumega OR oprelvekin OR factor xiii OR factor xiiia OR FXIII OR FXIIIa OR rFXIII OR rFXIIIa OR corifact OR fibrogammin OR tretten OR tranexamic OR aprotinin OR EACA OR aminocaproic) AND (haematological OR hematological OR haematology OR hematology OR haemato-oncology OR hemato-oncology OR bone marrow failure OR bone marrow disease OR bone marrow disorder OR leukemia OR leukaemia OR preleukemia OR preleukaemia OR aplastic OR hypoplastic OR refractory OR sideroblastic OR fanconi OR thrombocytopenia OR thrombocytopenic OR myelodysplasia OR bone marrow dysplasia OR myeloproliferative OR myelofibrosis OR fibrosis OR myelosclerosis OR shwachman OR dyskeratosis OR aml OR dysmyelopoiesis OR 5q syndrome) AND (randomized OR randomised OR randomly OR blind OR blinded OR trial OR control group OR groups))

\section{Appendix 9. KOREAMED search strategy}

\author{
"factor viia"[ALL] AND "Randomized Controlled Trial” [PT] \\ novoseven[ALL] AND “Randomized Controlled Trial” [PT]
}




\author{
fibrinogen[ALL] AND “Randomized Controlled Trial” [PT] \\ haemocomplettan[ALL] AND "Randomized Controlled Trial” [PT] \\ octafibrin[ALL] AND “Randomized Controlled Trial” [PT] \\ riastap[ALL] AND “Randomized Controlled Trial” [PT] \\ "platelet-poor plasma”[ALL] AND "Randomized Controlled Trial” [PT] \\ desmopressin[ALL] AND “Randomized Controlled Trial” [PT] \\ eltrombopag[ALL] AND "Randomized Controlled Trial” [PT] \\ promacta [ALL] AND "Randomized Controlled Trial” [PT] \\ revolade[ALL] AND “Randomized Controlled Trial” [PT] \\ romiplostim [ALL] AND "Randomized Controlled Trial” [PT] \\ nplate[ALL] AND “Randomized Controlled Trial” [PT] \\ thrombopoietin ALL] "Randomized Controlled Trial” [PT] \\ Interleukin[ALL] AND “Randomized Controlled Trial” [PT] \\ factor xiii[ALL] AND “Randomized Controlled Trial” [PT] \\ tranexamic [ALL] AND "Randomized Controlled Trial" [PT] \\ aprotinin [ALL] AND "Randomized Controlled Trial" [PT] \\ aminocaproic [ALL] AND "Randomized Controlled Trial” [PT] \\ EACA [ALL] AND “Randomized Controlled Trial” [PT] \\ antifibinolytic*[ALL] AND “Randomized Controlled Trial” [PT]
}

\title{
Appendix 10. Web of Science search strategy
}

Topic: (antifibrinolytics OR "factor viia" OR "factor 7a" OR rfviia OR fviia OR novoseven OR "novo seven" OR eptacog OR proconvertin OR "fibrinogen concentrate" OR "factor I" OR haemocomplettan OR octafibrin OR riastap OR "activated factor seven" OR "activated factor vii" OR "activated rfvii" OR "activated fvii" OR "factor seven" OR "factor vii" OR "factor 7" OR "platelet-poor plasma" OR desmopressin OR eltrombopag OR promacta OR revolade OR romiplastin OR romiplostim OR nplate OR "thrombopoietin receptor*" OR "thrombopoietin agonist*" OR "thrombopoietin mimetic*" OR "interleukin 6" OR “interleukin 11" OR IL-6 OR IL-11 OR sigosix OR neumega OR oprelvekin OR "factor xiii” OR FXIII OR rFXIII OR FXIIIa OR RFXIIIa OR corifact OR fibrogammin OR tretten OR tranexamic OR aprotinin OR EACA OR aminocaproic)

AND

Topic: (haematological OR hematological OR haematology OR hematology OR haematooncology OR hemato-oncology OR bone marrow failure OR bone marrow disease OR bone marrow disorder OR leukemia OR leukaemia OR preleukemia OR preleukaemia OR aplastic 
OR hypoplastic OR refractory OR sideroblastic OR fanconi OR thrombocytopenia OR thrombocytopenic OR myelodysplasia OR bone marrow dysplasia OR myeloproliferative OR myelofibrosis OR fibrosis OR myelosclerosis OR shwachman OR dyskeratosis OR AML OR dysmyelopoiesis OR 5q syndrome) AND

Topic: (systematic* OR random* OR blind* OR trial* OR control* OR groups)

\section{Appendix 11. ClinicalTrials.gov search strategy}

Search Terms: randomized OR randomised

Conditions: (hematological malignancies OR hemato-oncology OR bone marrow failure OR bone marrow disease OR leukemia OR preleukemia OR aplastic anemia OR hypoplastic anemia OR refractory anemia OR sideroblastic anemia OR fanconi OR thrombocytopenia OR myelodysplasia OR bone marrow dysplasia OR myeloproliferative OR myelofibrosis OR myelosclerosis OR shwachman OR dyskeratosis OR dysmyelopoiesis OR 5Q) AND

Interventions: (antifibrinolytics OR factor viia OR fibrinogen OR haemocomplettan OR platelet-poor plasma OR eltrombopag OR promacta OR revolade OR romiplastim OR romiplostim OR nplate OR thrombopoietin receptor agonist OR thrombopoietin agonist OR TPO OR interleukin 6 OR interleukin 11 OR IL-6 OR IL-11 OR sigosix OR neumega OR oprelvekin OR rhIL-11 OR factor xiii OR FXIII OR rFXIII OR corifact OR fibrogammin OR tretten OR tranexamic OR aprotinin OR EACA OR aminocaproic)

\section{Appendix 12. ICTRP search strategy}

Conditions: (marrow OR leukemia OR preleukemia OR anemia OR fanconi OR thrombocytopenia OR myelodysplasia OR hematological OR haematological OR hematooncological OR haemato-oncological OR myeloproliferative OR myelofibrosis OR myelosclerosis OR shwachman OR dyskeratosis OR dysmyelopoiesis)

AND

Intervention: (antifibrinolytics OR factor viia OR fviia OR rFViia OR novoseven OR desmopressin OR eptacog OR proconvertin OR fibrinogen concentrate OR haemocomplettan OR octafibrin OR riastap OR platelet-poor plasma OR eltrombopag OR promacta OR revolade OR romiplostim OR AMG531 OR AMG 531 OR thrombopoietin receptor agonist OR interleukin 6 OR interleukin 11 OR IL-6 OR IL-11 OR sigosix OR neumega OR oprelvekin OR rhIL-11 OR factor xiii OR FXIII OR rFXIII OR corifact OR fibrogammin OR tretten OR tranexamic OR aprotinin OR EACA OR aminocaproic)

\section{Appendix 13. Hong Kong Clinical Trials Registry search strategy}

Disease Group: Blood and blood-forming organs

Title: randomized OR randomised 


\section{Additional references}

Ades 2013 . Ades AE, Caldwell DM, Reken S, Welton NJ, Sutton AJ, Dias S. Evidence synthesis for decision making 7: a reviewer's checklist. Medical Decision Making. 2013; 33(5):679-91. [PubMed: 23804511]

Alter 2007 . Alter PA. Diagnosis, genetics, and management of inheritied bone marrow failure syndromes. Hematology (American Society of Hematology Education Program). 2007:29-32.

BCSH 2003 . British Committee for Standards in Haematology (BCSH). Guidelines for the use of platelet transfusions. British Journal of Haematology. 2003; 122(1):10-23. [PubMed: 12823341]

BCSH 2004 . British Committee for Standards in Haematology (BCSH). Transfusion guidelines for neonates and older children. British Journal of Haematology. 2004; 124(4):433-53. [PubMed: 14984493]

Chaimani 2012 . Chaimani A, Salanti G. Using network meta-analysis to evaluate the existence of small-study effects in a networkof interventions. Research Synthesis Methods. 2012; 3(2):16176. [PubMed: 26062088]

Chaimani 2013 . Chaimani A, Higgins JP, Mavridis D, Spyridonos P, Salanti G. Graphical tools for network meta-analysis in STATA. PLoS One. 2013; 8(10):e76654. [PubMed: 24098547]

Chaimani 2015 . Chaimani A, Salanti G. Visualizing assumptions and results in network metaanalysis: the network graphs package. The Stata Journal. 2015; 15(4):905-50.

Deeks 2011 . Deeks, JJ.; Higgins, JPT.; Altman, DG.; Higgins, JPT.; Green, S., editors. Cochrane Handbook for Systematic Reviews of Interventions Version 5.1.0 [updated March 2011]. The Cochrane Collaboration; 2011. Chapter 9: Analysing data and undertaking meta-analyses. Available from www.cochrane-handbook.org. The Cochrane Collaboration, Available from www.cochrane-handbook.org

Del Giovane 2013 . Del Giovane C, Vacchi L, Mavridis D, Filippini G, Salanti G. Network metaanalysis models to account for variability in treatment definitions: application to dose effects. Statistics in Medicine. 2013; 32(1):25-39. [PubMed: 22815277]

Desborough 2012 . Desborough M, Stanworth S. Plasma transfusion for bedside, radiologically guided, and operating room invasive procedures. Transfusion. 2012; 52(Suppl 1):20S-9S. [PubMed: 22578367]

Dinmohamed 2014 . Dinmohamed AG, Visser O, Van Norden Y, Huijgens PC, Sonneveld P, Van de Loosdrecht AA, et al. Trends in incidence, initial treatment and survival of myelodysplastic syndromes: a population-based study of 5144 patients diagnosed in the Netherlands from 2001 to 2010. European Journal of Cancer. 2014; 50(5):1004-12. [PubMed: 24388662]

Dokal 2008 . Dokal I, Vulliamy T. Inherited aplastic anaemias/bone marrow failure syndromes. Blood Reviews. 2008; 22(3):141-53. [PubMed: 18164793]

Estcourt 2011 . Estcourt LJ, Stanworth SJ, Murphy MF. Platelet transfusions for patients with haematological malignancies: who needs them? British Journal of Haematology. 2011; 154(4): 425-40. [PubMed: 21615375]

Estcourt 2014 . Estcourt LJ, Gregg R, Stanworth S, Doree C, Trivella M, Murphy MF, et al. Alternative agents versus prophylactic platelet transfusion for preventing bleeding in patients with haematological disorders after chemotherapy or stem cell transplantation. Cochrane Database of Systematic Reviews. 2014; (3)doi: 10.1002/14651858.CD010982

Foucar 1985 . Foucar K, Langdon RM, Armitage JO, Olson DB, Carroll TJ Jr. Myelodysplastic syndromes. A clinical and pathologic analysis of 109 cases. Cancer. 1985; 56(3):553-61. [PubMed: 3891071]

Franchini 2012 . Franchini M, Lippi G. Fibrinogen replacement therapy: a critical review of the literature. Blood Transfusion. 2012; 10(1):23-7. [PubMed: 22153684]

Gordon 1995 . Gordon MS, Nemunaitis J, Hoffman R, Paquette RL, Rosenfeld C, Manfreda S, et al. A phase I trial of recombinant human interleukin-6 in patients with myelodysplastic syndromes and thrombocytopenia. Blood. 1995; 85(11):3066-76. [PubMed: 7538815]

Gupta 1999 . Gupta P, LeRoy SC, Luikart SD, Bateman A, Morrison VA. Long-term blood product transfusion support for patients with myelodysplastic syndromes (MDS): cost analysis and complications. Leukemia Research. 1999; 23(10):953-9. [PubMed: 10573142] 
Heimpel 2000 . Heimpel, H. Epidemiology and aetiology of aplastic anaemia. In: Schrezenmeier, H.; Bacigalupo, A., editors. Aplastic Anaemia: Pathophysiology and Treatment. Cambridge University Press; Cambridge, UK: 2000. p. 97-116.

Higgins 2011a . Higgins, JPT.; Deeks, JJ.; Higgins, JPT.; Green, S., editors. Cochrane Handbook for Systematic Reviews of Interventions Version 5.1.0 [updated March 2011]. The Cochrane Collaboration; 2011. Chapter 7: Selecting studies and collecting data. Available from www.cochrane-handbook.org

Higgins 2011b . Higgins, JPT.; Altman, DG.; Sterne, JAC.; Higgins, JPT.; Green, S., editors. Cochrane Handbook for Systematic Reviews of Interventions Version 5.1.0 [updated March 2011]. The Cochrane Collaboration; 2011. Chapter 8: Assessing risk of bias in included studies. Available from www.cochrane-handbook.org

Higgins 2011c . Higgins, JPT.; Deeks, JJ.; Altman, DG.; Higgins, JPT.; Green, S., editors. Cochrane Handbook for Systematic Reviews of Interventions Version 5.1.0 [updated March 2011]. The Cochrane Collaboration; 2011. Chapter 16: Special topics in statistics. Available from www.cochrane-handbook.org

Higgins 2012 . Higgins JP, Jackson D, Barrett JK, Lu G, Ades AE, White IR. Consistency and inconsistency in network meta-analysis: concepts and models for multi-arm studies. Research Synthesis Methods. 2012; 3(2):98-110. [PubMed: 26062084]

Hutton 2015 . Hutton B, Salanti G, Caldwell DM, Chaimani A, Schmid CH, Cameron C, et al. The PRISMA extension statement for reporting of systematic reviews incorporating network metaanalyses of health care interventions: checklist and explanations. Annals of Internal Medicine. 2015; 162(11):777-7. [PubMed: 26030634]

Issaragrisil 2006 . Issaragrisil S, Kaufman D, Anderson T, Chansung K, Leaverton P, Shapiro S, et al. the Aplastic Anaemia Study Group. The epidemiology of aplastic anaemia in Thailand. Blood. 2006; 107(4):1299-307. [PubMed: 16254144]

Jackson 2014 . Jackson D, Barrett JK, Rice S, White IR, Higgins JP. A design-by-treatment interaction model for network meta-analysis with random inconsistency effects. Statistics in Medicine. 2014; 33(21):3639-54. [PubMed: 24777711]

Jansen 2013 . Jansen JP, Naci H. Is network meta-analysis as valid as standard pairwise metaanalysis? It all depends on the distribution of effect modifiers. BMC Medicine. 2013; 11:159. [PubMed: 23826681]

Kantarjian 2007 . Kantarjian H, Giles F, List A, Lyons R, Sekeres MA, Pierce S, et al. The incidence and impact of thrombocytopenia in myelodysplastic syndromes. Cancer. 2007; 109(9):1705-14. [PubMed: 17366593]

Karkouti 2013 . Karkouti K, Von Heymann C, Jespersen CM, Korte W, Levy JH, Ranucci M, et al. Efficacy and safety of recombinant factor XIII on reducing blood transfusions in cardiac surgery: a randomized, placebo-controlled, multicenter clinical trial. The Journal of Thoracic and Cardiovascular Surgery. 2013; 164(4):927-39. [PubMed: 23820174]

Konstantopoulos 1989 . Konstantopoulos K, Lauren L, Hast R, Reizenstein P. Survival, hospitalization and cause of death in 99 patients with the myelodysplastic syndrome. Anticancer Research. 1989; 9(4):893-6. [PubMed: 2817815]

Kurzrock 2001 . Kurzrock R, Cortes J, Thomas DA, Jeha S, Pilat S, Talpaz M. Pilot study of lowdose interleukin-11 in patients with bone marrow failure. Journal of Clinical Oncology. 2001; 19(21):4165-72. [PubMed: 11689585]

Kuter 2014 . Kuter DJ. Milestones in understanding platelet production: a historical overview. British Journal of Haematology. 2014; 165(2):248-58. [PubMed: 24528208]

Lefebvre 2011 . Lefebvre, C.; Manheimer, E.; Glanville, J.; Higgins, JPT.; Green, S., editors. Cochrane Handbook forSystematic Reviews of Interventions Version 5.1.0 [updated March 2011]. The Cochrane Collaboration; 2011. Chapter 6: Searching for studies. Available from www.cochrane-handbook.org. The Cochrane Collaboration

Lidbeck 1980 . Lidbeck J. Studies on hemopoietic dysplasia (the preleukemic syndrome). Clinical course and prognostic factors in 42 patients with dysplastic bone marrow. Acta Medica Scandinavica. 1980; 208(6):459-62. [PubMed: 7468314] 
Ma 2007 . Ma X, Does M, Raza A, Mayne ST. Myelodysplastic syndromes: incidence and survival in the United States. Cancer. 2007; 109(8):1536-42. [PubMed: 17345612]

Ma 2012 . Ma X. Epidemiology of myelodysplastic syndromes. The American Journal of Medicine. 2012; 125(7 Suppl):S2-5. [PubMed: 22735748]

Mannucci 1987 . Mannucci PM, Vicente V, Alberca I, Sacchi E, Longo G, Harris AS, et al. Intravenous and subcutaneous administration of desmopressin (DDAVP) to hemophiliacs: pharmacokinetics and factor VIII responses. Thrombosis and Haemostasis. 1987; 58(4):1037-9. [PubMed: 3127916]

Mavridis 2014 . Mavridis D, White IR, Higgins JP, Cipriani A, Salanti G. Allowing for uncertainty due to missing continuous outcome data in pairwise and network meta-analysis. Statistics in Medicine. 2014; 34(5):721-41. [PubMed: 25393541]

Montané 2008 . Montané E, Ibañez L, Vidal X, Ballarin E, Pig R, Garcia N, et al. the Catalan Group for the Study of Agranulocytosis and Aplastic Anemia. Epidemiology of aplastic anemia: a prospective multicenter study. Haematologica. 2008; 93(4):518-23. [PubMed: 18322256]

Muir 2003 . Muir KR, Chilvers CED, Harriss C, Coulson L, Grainge M, Darbyshire P, et al. The role of occupational and environmental exposures in the aetiology of acquired severe aplastic anaemia: a case control investigation. British Journal of Haematology. 2003; 123(5):906-14. [PubMed: 14632783]

NBA 2012 . National Blood Authority. The National Blood Authority's Patient Blood Management Guideline. National Blood Authority; Canberra: 2012. Module 3 - Medical.

Neukirchen 2011 . Neukirchen J, Schoonen WM, Strupp C, Gattermann N, Aul C, Haas R, et al. Incidence and prevalence of myelodysplastic syndromes: data from the Dusseldorf MDS-registry. Leukemia Research. 2011; 35(12):1591-6. [PubMed: 21708407]

NICE 2011 . NICE. Romiplostim for the treatment of chronic immune (idiopathic) thrombocytopenic purpura. National Institute of Health and Care Excellence; 2011. p. TA221

NICE 2013 . NICE. Eltrombopag for treating chronic immune (idiopathic) thrombocytopenic purpura (review of technology appraisal 205). National Institute of Health and Care Excellence; 2013. p. TA293

Parmar 1998 . Parmar MK, Torri V, Stewart L. Extracting summary statistics to perform metaanalyses of the published literature for survival endpoints. Statistics in Medicine. 1998; 17(24): 2815-34. [PubMed: 9921604]

Peters 2008 . Peters JL, Sutton AJ, Jones DR, Abrams KR, Rushton L. Contour-enhanced metaanalysis funnel plots help distinguish publication bias from other causes of asymmetry. Journal of Clinical Epidemiology. 2015; 61(10):991-6. [PubMed: 18538991]

Rauff 2011 . Rauff B, Idrees M, Shah SA, Butt S, Butt AM, Ali L, et al. Hepatitis associated aplastic anemia: a review. Virology Journal. 2011; 8:87. [PubMed: 21352606]

RevMan 2014 [Computer program] . The Nordic Cochrane Centre. The Cochrane Collaboration. Review Manager (RevMan). Version 5.3. The Nordic Cochrane Centre, The Cochrane Collaboration; Copenhagen: 2014.

Rhodes 2015 . Rhodes KM, Turner RM, Higgins JP. Predictive distributions were developed for the extent of heterogeneity in meta-analyses of continuous outcome data. Journal of Clinical Epidemiology. 2015; 68(1):52-60. [PubMed: 25304503]

Riley 2011 . Riley RD, Higgins JP, Deeks JJ. Interpretation of random effects meta-analyses. BMJ. 2011; 342:d549. [PubMed: 21310794]

Roberts 2013 . Roberts, DJ.; Prowse, CV. Blood substitutes. In: Murphy, MF.; Pamphilon, DH.; Heddle, NM., editors. Practical Transfusion Medicine. 4th Edition. Wiley-Blackwell; Chichester, UK: 2013. p. 399-409.

Ruggeri 2007 . Ruggeri ZM, Mendolicchio GL. Adhesion mechanisms in platelet function. Circulation Research. 2007; 100(12):1673-85. [PubMed: 17585075]

Salanti 2011 . Salanti G, Ades AE, Ioannidis JP. Graphical methods and numerical summaries for presenting results from multiple-treatment meta-analyses: an overview and tutorial. Journal of Clinical Epidemiology. 2011; 64(2):163-71. [PubMed: 20688472] 
Salanti 2012 . Salanti G. Indirect and mixed-treatment comparison, network, or multiple-treatments meta-analysis: many names, many benefits, many concerns for the next generation evidence synthesis tool. Research Synthesis Methods. 2012; 3(2):80-97. [PubMed: 26062083]

Salanti 2014 . Salanti G, Del Giovane C, Chaimani A, Caldwell DM, Higgins JP. Evaluating the quality of evidence from a network meta-analysis. PLoS One. 2014; 9(7):e99682. [PubMed: 24992266]

Schiffer 2001 . Schiffer CA, Anderson KC, Bennett CL, Bernstein S, Elting LS, Goldsmith M, et al. Platelet transfusion for patients with cancer: Clinical practice guidelines of the American Society of Clinical Oncology. Journal of Clinical Oncology. 2001; 19(5):1519-38. [PubMed: 11230498]

Schünemann 2011 . Schünemann, HJ.; Oxman, AD.; Higgins, JPT.; Vist, GE.; Glasziou, P.; Guyatt, GH.; Higgins, JPT.; Green, S., editors. Cochrane Handbook for Systematic Reviews of Interventions Version 5.1.0 [updated March 2011]. The Cochrane Collaboration; 2011. Chapter 11: Presenting results and 'Summary of findings' tables. Available from www.cochranehandbook.org

Simpson 2012 . Simpson E, Lin Y, Stanworth S, Birchall J, Doree C, Hyde C. Recombinant factor VIIa for the prevention and treatment of bleeding in patients without haemophilia. Cochrane Database of Systematic Reviews. 2012; (3)doi: 10.1002/14651858.CD005011.pub4

Slichter 2007 . Slichter SJ. Evidence-based platelet transfusion guidelines. Hematology (American Society of Hematology Education Program). 2007:172-8.

StataCorp 2011 [Computer program] . StataCorp.. Stata Statistical Software. Release 12. StataCorp; College Station, TX: 2012.

Sterne 2011 . Sterne, JAC.; Egger, M.; Moher, D.; Higgins, JPT.; Green, S., editors. Cochrane Handbook for Systematic Reviews of Interventions. Version 5.1.0 (updated March 2011). The Cochrane Collaboration; Chapter 10: Addressing reporting biases. Available from www.cochrane-handbook.org, 2011

Svensson 2014 . Svensson PJ, Bergqvist PBF, Juul KV, Berntorp E. Desmopressin in treatment of haematological disorders and in prevention of surgical bleeding. Blood Reviews. 2014; 28(3):95102. [PubMed: 24703870]

Swift 1971 . Swift M. Fanconi's anaemia in the genetics of neoplasia. Nature. 1971; 230(5293):3703. [PubMed: 4927726]

Tefferi 2013 . Tefferi A. Primary myelofibrosis: 2013 update on diagnosis, risk-stratification, and management. American Journal of Hematology. 2013; 88(2):141-50. [PubMed: 23349007]

Tierney 2007 . Tierney JF, Stewart LA, Ghersi D, Burdett S, Sydes MR. Practical methods for incorporating summary time-to-event data into meta-analysis. Trials. 2007; 8:16. [PubMed: 17555582]

Tinmouth 2007 . Tinmouth, AT. Canadian Blood Services. 4th Edition. Canadian Blood Services; 2007. Chapter 18: Platelet transfusion, alloimmunization and management of platelet refractoriness.

Titmarsh 2014 . Titmarsh GJ, Duncombe AS, McMullin MF, O’Rorke M, Mesa R, De Vocht F, et al. How common are myeloproliferative neoplasms? A systematic review and meta-analysis. American Journal of Hematology. 2014; 89(6):581-7. [PubMed: 24971434]

Tsimberidou 2005 . Tsimberidou AM, Giles FJ, Khouri I, Bueso-Ramos C, Pilat S, Thomas DA, et al. Low-dose interleukin-11 in patients with bone marrow failure: update of the M. D. Anderson Cancer Center experience. Annals of Oncology. 2005; 16(1):139-45. [PubMed: 15598951]

Turner 2012 . Turner RM, Davey J, Clarke MJ, Thompson SG, Higgins JP. Predicting the extent of heterogeneity in meta-analysis, using empirical data from the Cochrane Database of Systematic Reviews. International Journal of Epidemiology. 2012; 41(3):818-27. [PubMed: 22461129]

Verma 2009 . Verma A, Agarwal P. Platelet utilization in the developing world: strategies to optimize platelet transfusion practices. Transfusion and Apheresis Science. 2009; 41(2):145-9. [PubMed: 19716339]

Wardrop 2013 . Wardrop D, Estcourt L, Doree C, Hopewell S, Stanworth S, Murphy MF. Antifibrinolytics (lysine analogues) for the prevention of bleeding in patients with haematological disorders. Cochrane Database of Systematic Reviews. 2013; (7)doi: 10.1002/14651858.CD009733.pub2 
White 2008 . White IR, Higgins JP, Wood AM. Allowing for uncertainty due to missing data in meta-analysis-part 1: two-stage methods. Statistics in Medicine. 2008; 27(5):711-27. [PubMed: 17703496]

White 2011 . White I. Multivariate meta-analysis: updates to mvmeta. The Stata Journal. 2011; 11(2):255-70.

White 2012 . White IR, Barrett JK, Jackson D, Higgins JP. Consistency and inconsistency in network meta-analysis: model estimation using multivariate meta-regression. Research Synthesis Methods. 2012; 3(2):111-25. [PubMed: 26062085]

White 2015 . White I. Network meta-analysis. Stata Journal. 2015; 15(4):951-985.

Wikkelsø 2013 . Wikkelsø A, Lunde J, Johansen M, Stensballe J, Wetterslev J, Møller AM, et al. Fibrinogen concentrate in bleeding patients. Cochrane Database of Systematic Reviews. 2013; (8)doi: 10.1002/14651858.CD008864.pub2

Young 2008 . Young NS, Kaufman DW. The epidemiology of acquired aplastic anemia. Haematologica. 2008; 93(4):489-92. [PubMed: 18379007]

Zeng 2011 . Zeng Y, Duan X, Xu J, Ni X. TPO receptor agonist for chronic idiopathic thrombocytopenic purpura. Cochrane Database of Systematic Reviews. 2011; (7)doi: 10.1002/14651858.CD008235.pub2

* Indicates the major publication for the study 


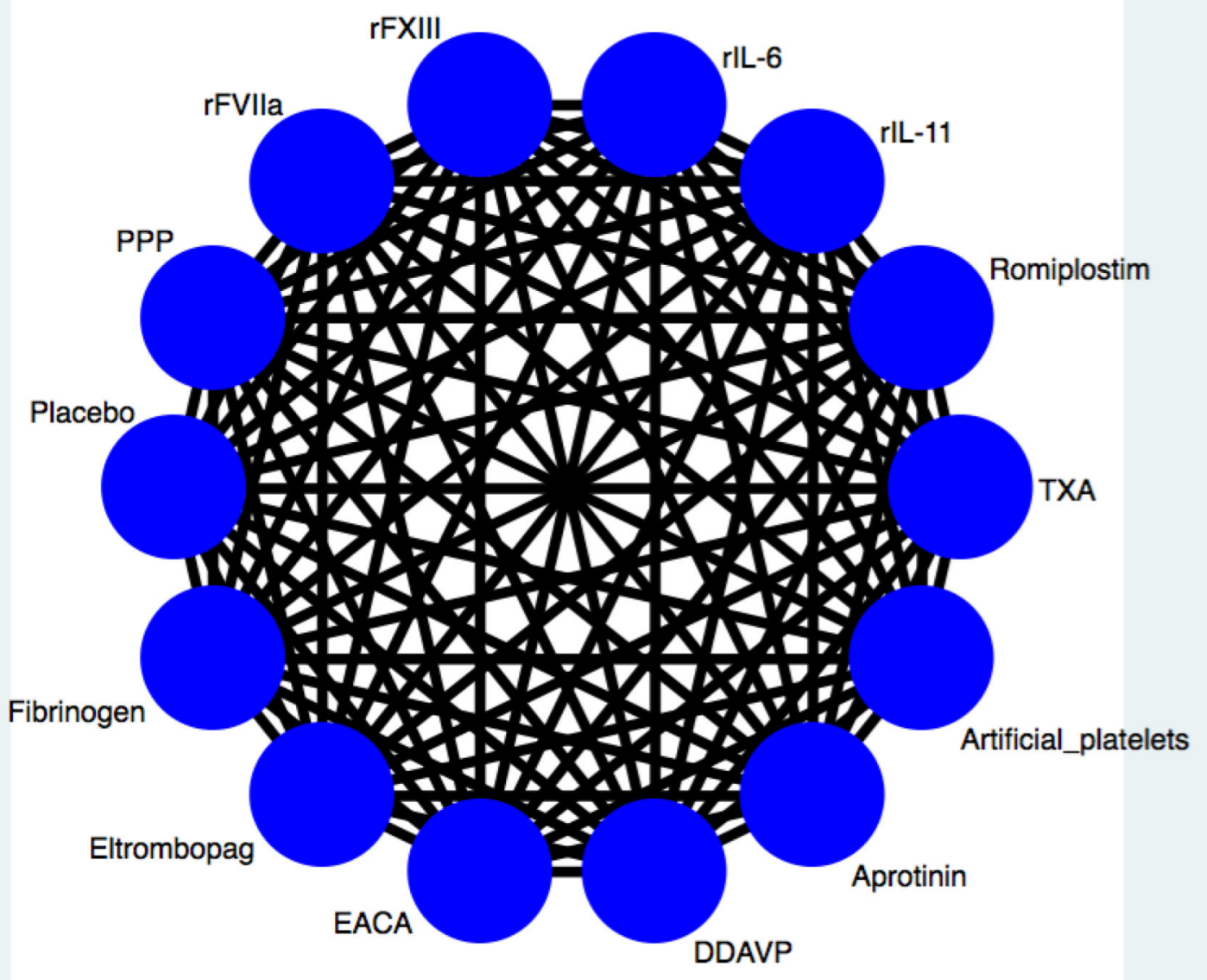

Figure 1 . Network graph demonstrating all possible pairwise comparisons. DDAVP desmopressin, EACA - epsilon aminocaproic acid, PPP - platelet poor plasma, rFVIIa recombinant activated factor VII, rFXIII - recombinant factor XIII, rIL-6 - recombinant interleukin 6, IL-11 - recombinant interleukin 11 and TXA - tranexamic acid 